\title{
Numerical investigation of cable breakage events on long-span cable-stayed bridges under stochastic traffic and wind
}

Yufen Zhou $^{1}$ and Suren Chen ${ }^{2^{\star}}$

\section{Abstract}

Cable breakage (loss) events can be disastrous to cable-stayed bridges because of potential risks of progressive collapse following the initial failure of stay cables. To avoid turning a cable-breakage hazard into a disaster, it is important to rationally assess the risk through accurately predicting the nonlinear dynamic behavior of the bridge subjected to various types of cable-loss events. With a recently developed advanced finite element (FE)-based nonlinear dynamic simulation platform, a comprehensive numerical investigation of cable-loss incidents on a long-span cable-stayed bridge is conducted by focusing on postbreakage performance. Parametric studies are carried out to evaluate the impacts from various important parameters related to cable-breakage process and service loads from stochastic traffic and wind. Several parameters associated with cable-breakage processes, such as the breakage duration, time-transient curve and initial state, are found to influence the dynamic performance of the bridge notably. The results also indicate that service traffic and wind loads as well as complex coupling effects with the bridge are important to the bridge response following cable-loss events. In the final part of the study, response envelope analysis is made and a comparative investigation is also conducted between the results from the advanced FE-based nonlinear dynamic approach and those from the equivalent static approach as suggested by the Post-Tensioning Institute (PTI).

Keywords: Cable-stayed bridge; long-span bridge; cable breakage (loss); wind; traffic

\footnotetext{
${ }^{1}$ Ph.D. Candidate, Dept. of Civil \& Environmental Engineering, Colorado State University, Fort Collins, CO 80523, Email: yufzhou@rams.colostate.edu.

$2{ }^{*}$ Corresponding author. P.E., Associate Professor, Dept. of Civil \& Environmental Engineering, Colorado State University, Fort Collins, CO 80523, Email: suren.chen@colostate.edu, tel: 970-491-7722, fax: 970-491-7727.
} 


\section{Introduction}

23 Although the total number of long-span bridges is much smaller than that of short-span and medium-span

24 bridges in the world, long-span bridges have attracted a lot of attention from engineers and general public.

25 As a critical linkage of key transportation corridors and even evacuation routes, the failure of a long-span

26 bridge like I-35W Bridge in Minnesota has significant impacts on local transportation, economy and

27 many other aspects of the whole society (NTSB 2007). Due to the intrinsic complexity of structures, the

28 loads and related uncertainties, a reliable prediction of lifetime performance of long-span bridges still

29 needs to be further explored (ASCE 2003). It is underscored in a special report about existing bridges by

30 ASCE (2003) that “... Although some performance aspects of our long-span bridges have been already

31 tested through long-term usage, extreme environmental conditions, extreme natural events, or by unusual

32 man-made accidents or incidents (collisions, fire, explosions, blasts etc.), these represent only a small

33 percentage of the total credible events that must be investigated and assessed for the continued lifetime

34 performance of these bridges."

35 In addition to sharing common complexities and challenges that most long-span bridges experience, cable-stayed long-span bridges also face their unique threats such as cable loss (breakage). Different from the common understanding that cable-stayed bridges have high intrinsic redundancy of stayed cables, studies on the behavior of cables subjected to high velocity impact suggest that abrupt cable loss is readily achievable, particularly under intentional attack scenarios (e. g. Zoli and Woodward 2005). Within the past ten years, a number of important studies have been carried out about cable loss on cable-stayed bridges. Being able to more accurately investigate the dynamic response of the bridge following a cable-

42 loss incident, nonlinear dynamic analysis has been commonly adopted in recent years (e.g., Ruiz-Teran 43 and Aparicio 2009; Mozos and Aparicio 2010a \& 2010b). Ruiz-Teran and Aparicio (2009) conducted 44 detailed parametric studies with respect to cable breakage duration, application of breakage load, and type of deviator, etc. In addition to numerical simulations, Mozos and Aparicio (2011) also experimentally investigated the impact of cable breakage duration on the dynamic response of a cable structure and the

47 load-time relation in the cable breakage process. Recently, Cai et al. (2012) and Wolff and Starossek 
proposed a nonlinear dynamic analysis methodology for long-span cable-stayed bridges with the commercial finite element (FE) software SAP2000 to simulate the cable breakage scenarios under no or very mild wind conditions. All the existing simulation methodologies so far, however, were not applicable to long-span cable-stayed bridges experiencing complex dynamic interactions originating from the excitations from both wind and stochastic traffic. As a result, some more insightful observations of cable-loss incidents of long-span cable-stayed bridges considering both wind and traffic are not yet available. dynamic simulation platform for breakage of stay cables was proposed with several important improvements over the existing simulation techniques. These include improved modeling of cable-loss process, consideration of realistic wind and traffic loads through the complex bridge-traffic-wind interaction model on a finite-element (FE) basis and incorporation of geometric and material nonlinearities from various sources. Such a platform offers so far the most advanced and versatile simulation tool applicable to various kinds of cable-stayed bridges, including those slender ones susceptible to wind. The objective of the present study is to carry out a comprehensive investigation of cable-loss phenomena with the recently developed platform to improve the knowledge about various cable-loss events and their impacts on bridge safety. Specifically, a comprehensive parametric investigation on a prototype long-span cable-stayed bridge is carried out to study the impact on the post-

67 breakage bridge response from cable-breakage parameters such as cable-breakage process, duration and 68 initial state. The influences of dynamic excitations from stochastic traffic and wind as well as the 69 coupling effects on the post-breakage response of the bridge are also investigated. Response envelopes on 70 the bridge girders, pylons and stay cables due to breakage of single stay cable are obtained using the 71 nonlinear dynamic simulation methodology in the present study and then compared to those obtained with 72 the equivalent static analysis approach as introduced by the Post-Tensioning Institute (PTI) in order to 73 shed some lights on the applicability of the popular codified analytical approach on long-span bridges. 


\section{Nonlinear dynamic simulation methodology}

75 The nonlinear dynamic simulation methodology, as the key part of the FE-based nonlinear dynamic

76 simulation platform, is briefly introduced in the following to provide essential background for the present

77 study and more details about the platform can be found in Ref (Zhou and Chen 2014b; Zhou and Chen 2015). The simulation framework is coded with MATLAB programming language (MATLAB R2013b).

\subsection{The fully-coupled bridge-traffic-wind interaction analysis}

\subsubsection{Stochastic traffic flow simulation}

81 Stochastic traffic flow is simulated to represent the realistic vehicle motion on the bridge when cable breakage occurs (Chen and $\mathrm{Wu} 2010,2011)$. The instantaneous behavior of vehicles in the traffic flow is computationally efficient microscopic simulation methodology in the sense that time advances in discrete steps and space is discretized into multiple cells, each of which is empty or occupied with one vehicle. By applying a set of probabilistic traffic transition rules that regulate the accelerating, decelerating, lane changing and braking, the discrete variables for each cell at any time are updated based on the vehicle information in the adjacent cells (Chen and Wu 2011).

\subsubsection{Structural idealization and finite element modeling of the bridge}

The cable-stayed bridge system is established as a three-dimensional finite element model in this study by using two types of elements. The bridge superstructure and pylons are modeled with nonlinear

92 three-dimensional spatial beam elements based on the Timoshenko beam theory in which the axial, 93 bending, torsional warping and shear deformation effects are considered at the same time. The stay cables

94 are modeled as two-node catenary cable elements based on the analytical explicit solution that is obtained

95 from the differential equations and boundary conditions for a cable with elastic catenary.

\section{$96 \quad 2.1 .3 \quad$ Modeling of road vehicles}

97 Typical vehicles in the traffic flow are categorized into three types, which are heavy trucks, light 
several rigid bodies and wheel axles connected by series of springs, dampers and pivots. The upper and

100 lower springs are used to model the suspension system of each axle and elastic tires, respectively. Viscous

101 dampers are used to model the energy dissipation system. The mass of the suspension system is assumed

102 to be concentrated on the secondary rigid body at each wheel axle while the springs and dampers are

103 assumed massless. The heavy truck model has two rigid bodies and three wheel axles, which are

104 connected by 24 sets of springs and dampers. The displacement vector for the heavy truck model has 19

105 Degree-of-Freedoms (DOFs), consisting of 8 independent vertical, 8 lateral and 3 rotational DOFs. The

106 vehicle model for light truck and light car has a single rigid body and two wheel axles, connected by 16

107 sets of springs and dampers. The displacement vector for light truck and light car includes 5 independent

108 vertical, 5 lateral and 2 torsional DOFs. The elevation and front views for the heavy truck are shown in

109 Figs. 1a and b, respectively.

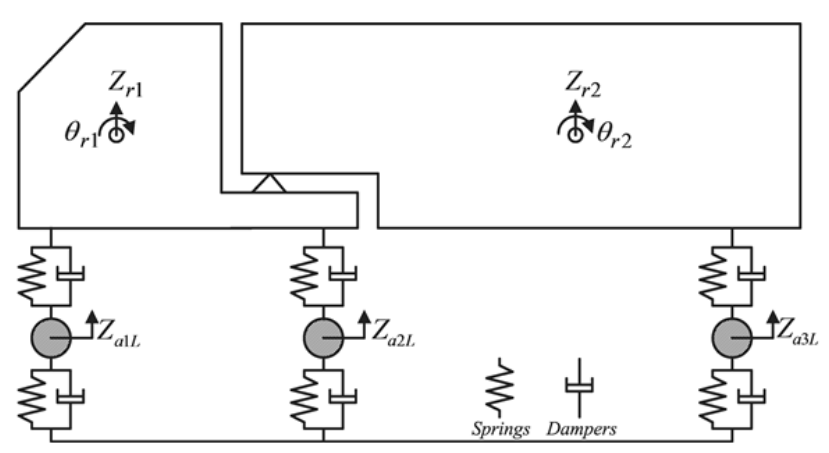

(a) Elevation view of vehicle

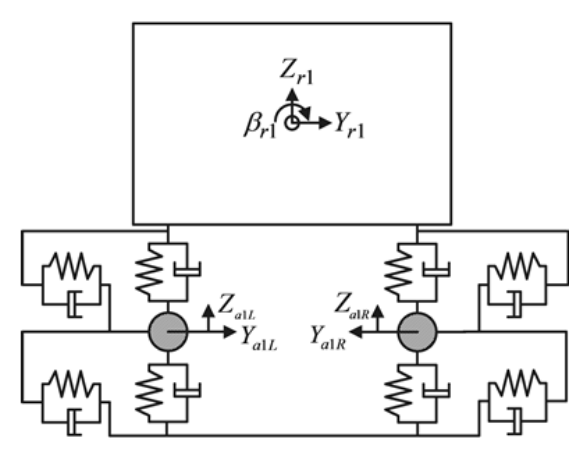

(b) Front view of vehicle

Fig. 1 Vehicle model for heavy truck

\section{$111 \quad$ 2.1.4 Modeling of wind forces on the bridge}

112 The wind forces acting on the bridge girder are commonly separated into three components: steady-

113 state forces resulting from mean wind speed, self-excited forces resulting from wind-bridge interactions

114 and buffeting forces resulting from unsteady wind velocity. The wind forces are usually discretized as lift

115 force, drag force and torsional moment for each of the three wind force components acting on bridge 116 girders. For bridge pylons and stay cables, only the drag component of the steady-state wind force 
117 corresponding to initial wind attack angle is considered. For bridge girders, the lift force is taken as the 118 example to demonstrate the steady-state, self-excited and buffeting force components.

119 The steady-state lift force on a unit span of the bridge girder is expressed in Eq. (1).

$$
L_{s t}=\frac{1}{2} \rho U_{w}^{2} C_{l}(\alpha) H
$$

121 in which, $\rho$ is the mass density of air; $U_{w}$ is the steady-state wind speed; $\alpha$ is the effective wind attack 122 angle which is obtained by summing initial wind attack angle and the rotational displacement of the 123 bridge girder in the nonlinear static analysis; $C_{l}$ is the lift force coefficient, which is a function of the 124 effective wind attack angle $\alpha ; H$ is the height of the cross section of the bridge girder.

125 The self-excited lift force on a unit span of the bridge girder can be expressed by the summation of 126 the convolution integrals between the arbitrary bridge girder motion and the associated impulse functions, 127 as shown in Eq. (2)

$$
L_{s e}(t)=L_{s e p}(t)+L_{s e h}(t)+L_{s e \alpha}(t)=\int_{-\infty}^{t} f_{L p}(t-\tau) p(\tau) d \tau+\int_{-\infty}^{t} f_{L h}(t-\tau) h(\tau) d \tau+\int_{-\infty}^{t} f_{L \alpha}(t-\tau) \alpha(\tau) d \tau
$$

129 in which, $f$ is the response impulse functions; $p(\tau), h(\tau)$ and $\alpha(\tau)$ are the lateral, vertical and rotational 130 displacements of the bridge girder with respect to time; the subscript " $L$ " refers to the response 131 contribution to the lift self-excited force; the subscripts " $p$ ", " $h$ " and " $\alpha$ " indicate impulse response 132 functions with respect to unit impulse displacements in the lateral, vertical and torsional directions, 133 respectively.

134 The buffeting lift force can be formulated using similar method for self-excited lift force, as shown in 135 Eq. (3).

$$
L_{b}(t)=L_{b u}(t)+L_{b w}(t)=\int_{-\infty}^{t} f_{L u}(t-\tau) u(\tau) d \tau+\int_{-\infty}^{t} f_{L w}(t-\tau) w(\tau) d \tau
$$

137 in which, the subscripts " $u$ " and " $w$ " represent turbulent wind horizontal and vertical velocities, 138 respectively. 


\subsubsection{Modeling of wind forces on road vehicles}

140 Different from the way of separating the total wind forces into steady and unsteady components on the

141 bridge, the total wind force is applied on the vehicles using a quasi-static approach proposed by Baker

142 (1986). In this study the total wind velocity is assumed to be perpendicular to the longitudinal direction of

143 the bridge girder. The relative wind velocity $U_{R}$ to a vehicle driven on the bridge can be expressed in the

144 following equation.

$$
U_{R}(t)=\sqrt{\left(U_{w}+u(x, t)\right)^{2}+U_{v e}^{2}(t)}
$$

146 in which, $u(x, t)$ is the turbulent wind speed.

147 The aerodynamic forces and moments on the vehicles have six components, which are drag force, 148 side force, lift force, rolling moment, pitching moment and yawing moment. Taking the lift force for 149 instance, the lift wind force on the vehicle is expressed in Eq. (5a).

$$
F_{v z}^{t}(t)=\frac{1}{2} \rho U_{R}^{2}(t) C_{L}(\Psi) A
$$

151 in which, $C_{L}(\Psi)$ is the lift force coefficient and $A$ is the reference area. $\psi$ is the yaw angle, which is the 152 angle between the direction of relative wind speed and the vehicle driving direction in the range from 0 to $153 \pi$, as defined in Eq. (5b):

$$
\psi(t)=\arctan \left[U_{w}+u(x, t) / U_{v e}(t)\right]
$$

\subsubsection{Equations of motion for the fully-coupled bridge-traffic-wind system}

156 The coupled dynamic motion equations of the bridge-traffic system can be expressed as:

$$
\left[\begin{array}{cccc}
M_{b} & 0 & \cdots & 0 \\
0 & M_{v_{1}} & \cdots & 0 \\
\vdots & \vdots & \ddots & \vdots \\
0 & 0 & \cdots & M_{v_{n}}
\end{array}\right]\left\{\begin{array}{c}
\ddot{q}_{b} \\
\ddot{q}_{v_{1}} \\
\vdots \\
\ddot{q}_{v_{n}}
\end{array}\right]+\left[\begin{array}{cccc}
C_{b} & 0 & \cdots & 0 \\
0 & C_{v_{1}} & \cdots & 0 \\
\vdots & \vdots & \ddots & \vdots \\
0 & 0 & 0 & C_{v_{n}}
\end{array}\right]\left[\begin{array}{c}
\dot{q}_{b} \\
\dot{q}_{v_{1}} \\
\vdots \\
\dot{q}_{v_{n}}
\end{array}\right]+\left[\begin{array}{cccc}
K_{b} & 0 & \cdots & 0 \\
0 & K_{v_{1}} & \cdots & 0 \\
\vdots & \vdots & \ddots & \vdots \\
0 & 0 & \cdots & K_{v_{n}}
\end{array}\right]\left\{\begin{array}{c}
q_{b} \\
q_{v_{1}} \\
\vdots \\
q_{v_{n}}
\end{array}\right\}=\left\{\begin{array}{c}
\sum_{i=1}^{n} F_{v_{i}}^{G}+F_{b}{ }^{R}+F_{b}^{C}+F_{b}{ }^{S e}+F_{b}{ }^{B u} \\
F_{v_{1}}{ }^{2}+F_{v_{1}}{ }^{C}+F_{v_{1}}{ }^{d w} \\
\vdots \\
F_{v_{n}}{ }^{R}+F_{v_{n}}{ }^{C}+F_{v_{n}}{ }^{d w}
\end{array}\right\}
$$

in which, $n$ is the total number of vehicles; $q$ is the displacement vector; $M, K, C$ and $F$ are structural mass, stiffness, damping matrices and force vector; subscripts $b$ and $v_{\mathrm{i}}(i=1,2, \ldots, n)$ indicate the

160 parameters for the bridge and the $i^{\text {th }}$ vehicle, respectively; the superscripts $G, R, C, W, S e$ and $B u$ refer to 
161 the external loads from gravity, road surface roughness, coupling interaction forces, static wind, self-

162 excited and buffeting forces, respectively.

163 The coupled forces on the bridge and the $i^{\text {th }}$ vehicle are defined in Eqs. (6b) and (6c), respectively.

$$
F_{b}^{C}=\sum_{i=1}^{n} C_{b c i}\left\{\dot{q}_{b}\right\}+\sum_{i=1}^{n} K_{b c i}\left\{q_{b}\right\}+\sum_{i=1}^{n} C_{b, v_{i}}\left\{\dot{q}_{v_{i}}\right\}+\sum_{i=1}^{n} K_{b, v_{i}}\left\{q_{v_{i}}\right\}
$$

$$
F_{v_{i}}{ }^{C}=C_{v_{i}, b}\left\{\dot{q}_{v_{i}}\right\}+K_{v_{i}, b}\left\{q_{v_{i}}\right\}
$$

in which, $K_{b c i}$ and $C_{b c i}$ refer to the stiffness and damping contribution to the bridge structure due to the

168 coupling effects between the $i^{\text {th }}$ vehicle in the traffic flow and the bridge system, respectively; $K_{b, v_{i}}\left(K_{v_{i}, b}\right)$

169 and $C_{b, v_{i}}\left(C_{v_{i}, b}\right)$ are the coupled stiffness and damping matrices between the bridge and the $i^{\text {th }}$ vehicle's

170 vibration, respectively.

171 The coupling matrices are formulated based on the instantaneous contacting locations of the vehicle

172 wheels on the bridge, which therefore need to be updated at each time step. For the motion-dependent

173 forces involved in the analysis, such as the self-excited forces due to turbulent wind speeds and coupling

174 interaction forces between the bridge and vehicles, nonlinear iteration is conducted to obtain the dynamic

175 response of the bridge-traffic system starting with an initial motion vector at each time step. The iteration

176 process at each time step will terminate when the prescribed convergence criterion is met.

\section{$177 \quad 2.2$ Nonlinear dynamic simulation strategy for cable breakage scenario}

\section{$178 \quad 2.2 .1 \quad$ Nonlinearity involved in the analysis}

\subsubsection{Geometric nonlinearity}

180 By formulating the equilibrium equations for a catenary cable, various sources of geometric 181 nonlinearity of the cable elements are incorporated in the simulation process, including cable sag effect,

182 large displacement effect and tension-stiffening effect. The geometric stiffness of the beam elements on 183 the bridge girders and pylons are considered in the tangent stiffness. 


\subsubsection{Material nonlinearity}

185 The concentrated plastic hinge approach is applied to consider the material nonlinearity of the beam 186 and cable elements, which may be incurred during the cable breakage simulation process. The yielding 187 state of the beam element is determined according to the prescribed interacting yielding surface of axial 188 force and bi-axial bending moments at the hinge location. The plastic hinge for the beam element is 189 defined as $P M M$ hinge, named for the three components forming the interacting yielding surface, which 190 include axial force $P$ and two bending moments $M M$ around the two orthogonal axes of the cross section.

191 The plastic tangent stiffness matrix of the beam element $K_{p l}$ is expressed in Eq. (7).

$$
K_{p l}=K_{e}-K_{e} N\left(N^{t}\left(K_{p}+K_{e}\right) N\right)^{-1} N^{t} K_{e}
$$

193 in which, $K_{e}$ is the elastic tangent stiffness matrix; $K_{p}$ is the plastic stiffness matrix, which depends on the 194 hardening slope of the element stiffness; $N$ is the gradient matrix of the yield surface function. The 195 interaction yielding surface $\Psi$ is conservatively selected as an octahedron surface, which can be expressed 196 in the following equation.

$$
\Psi\left(P, M_{y}, M_{z}\right)=\frac{|P|}{P_{\text {yield }}}+\frac{\left|M_{y}\right|}{M_{\text {yyield }}}+\frac{\left|M_{z}\right|}{M_{\text {zyield }}}=1
$$

198 in which, $P, M_{y}$ and $M_{z}$ are the axial force, bending moment around $y$-axis and $z$-axis of the member,

199 respectively; $P_{\text {yield }}, M_{y y i e l d}$ and $M_{z y i e l d}$ are the axial force, bending moment around $y$-axis and $z$-axis of the 200 member at yield state, respectively; $P_{\text {yield }}=f_{y} \cdot A ; M_{\text {yyield }}=f_{y} \cdot S_{p y} ; M_{\text {zyield }}=f_{y} \cdot S_{p z} ; f_{y}$ is the yield strength;

$201 A$ is the sectional area; $S_{p y}$ and $S_{p z}$ are the plastic sectional modulus around $y$-axis and $z$-axis of the 202 member cross section, respectively.

203 Different from the interacting $P M M$ hinge for beam elements, the plastic hinge model for cable 204 elements can only bear pure axial forces and is thus called $P$ hinge, indicating only axial force $P$ is used to 205 determine the yielding state. The yielding state $\Omega$ for cable elements is expressed in the following 206 equation.

$$
\Omega(P)=\frac{|P|}{P_{\text {yield }}}=1
$$


Considering a cable breakage event starting at $t_{1}$ second in the total simulation duration of $\left(t_{1}+t_{2}\right)$ seconds, the cable-loss simulation procedure is described as follows:

211 Step 1: Obtain the initial deformed displacement vector of the bridge $\left\{d_{s b}\right\}$ from nonlinear static 212 analysis of the bridge system under the bridge self-weight and static wind load. For vehicles, the 213 displacement is measured from the equilibrium position under vehicle self-weight. The initial 214 displacement vector $\left\{d_{s v}\right\}$ for all the vehicles is zero.

215 Step 2: Obtain the nodal axial forces of the cable to fail at $t_{1}$ second, which are denoted as $F_{b 1}$ and $F_{b 2}$ 216 for the upper and lower ends of the cable, respectively, from nonlinear dynamic analysis of the bridge217 traffic system starting from $\left\{d_{s b}\right\}$ and $\left\{d_{s v}\right\}$.

218 Step 3: Obtain the nodal forces $F_{b 1 t}$ and $F_{b 2 t}$ and apply them to the end nodes of the breaking cable at 219 current time step.

220 Step 3 can be further decomposed into five sub-steps.

221 Sub-step 3-1: Determine the relative breakage time $t_{r e l}$ as defined in Eq. (10) as a ratio between 222 the elapsed breakage time $t_{\text {elap }}$ after the breakage starts and the total breakage duration $t_{\text {total }}$.

$$
t_{\text {rel }}=t_{\text {elap }} / t_{\text {total }}
$$

Sub-step 3-2: Determine the relative loss of cable $l_{\text {rel }}$ at current time step from the breakage process function $f$.

$$
l_{\text {rel }}=f\left(t_{\text {rel }}\right)
$$

Sub-step 3-3: Update the current effective area of cable $A_{\text {eff }}$ for the failure in the global structural formulation using Eq. (12).

$$
A_{\text {eff }}=\left(1-l_{\text {rel }}\right) \cdot A_{\text {orig }}
$$

230 in which, $A_{\text {orig }}$ is the original cable area of the breaking cable.

$$
F_{b 1(2) t}=l_{r e l} \cdot F_{b 1(2)}
$$


Sub-step 3-5: Apply the nodal forces $F_{b 1 t}$ and $F_{b 2 t}$ with the same amplitude but in the opposite directions to the two anchorage nodes of the cable for failure at time instant $t_{1}+t_{\text {elap }}$. another $t_{2}$ seconds.

During the simulation process in each time step, every bridge member will be evaluated at each iteration step in terms of whether it may get into the inelastic range or fail. When all the members behave

241 linearly, the structural matrices will be updated considering the geometric nonlinearity and the 242 nonlinearity associated with bridge-traffic and bridge-wind interactions. For the members that yield 243 without failing, the element tangent stiffness matrix will be reduced to the plastic stiffness matrix and the 244 fixed end forces will also be updated. The members that meet the prescribed failure criterion will be 245 eliminated from the bridge FE model.

\section{Parametric Analysis of Single-cable Breakage Scenarios}

As discussed in the previous section, a rational characterization of cable-loss events involves the uncertainties about cable breakage, such as duration, time-transient processes, and the time instant of occurrence. Besides, various possible combinations of service loads for different cable-breakage events 250 present another challenge. In order to better understand the nature of cable-loss, a parametric study is 251 conducted for these variables associated with single-cable breakage events. To facilitate the readers 252 understanding the following sections, a 3-D sketch of the prototype bridge is shown in Fig. 2. The 253 mechanical and sectional parameters are given in Table 1 and Table 2, respectively. The three lowest

254 frequencies of the bridge are $0.405,0.652$ and $0.682 \mathrm{~Hz}$, which correspond to the symmetric vertical, 255 symmetric lateral and anti-symmetric vertical mode shapes, respectively. 
256

257

258

\begin{tabular}{cccc}
\hline Properties & Unit & Girders and pylons & Stay cables \\
\hline Weight density, $\gamma$ & $\mathrm{kN} / \mathrm{m}^{3}$ & 76.97 & 74.86 \\
Elasticity modulus, $E$ & $\mathrm{kN} / \mathrm{m}^{2}$ & $2.0 \mathrm{E}+8$ & $2.0 \mathrm{E}+8$ \\
Poisson's ratio, $\rho$ & - & 0.30 & 0.27 \\
Shear modulus, $G$ & $\mathrm{kN} / \mathrm{m}^{2}$ & $7.69 \mathrm{E}+7$ & $7.87 \mathrm{E}+7$ \\
Yield stress, $F_{y}$ & $\mathrm{kN} / \mathrm{m}^{2}$ & $2.48 \mathrm{E}+5$ & $1.49 \mathrm{E}+6$ \\
Ultimate stress, $F_{u}$ & $\mathrm{kN} / \mathrm{m}^{2}$ & $3.45 \mathrm{E}+5$ & $1.72 \mathrm{E}+6$ \\
\hline
\end{tabular}

Fig. 2 3-D sketch of the prototype long-span cable-stayed bridge

Table 1 Mechanical properties of the bridge

Table 2 Sectional properties of the bridge

\begin{tabular}{cccc}
\hline Properties & Unit & Girders & Pylons \\
\hline Vertical moment of inertia, $I_{y}$ & $m^{4}$ & 1.548 & $0.687 \sim 3.532$ \\
Vertical plastic modulus, $S_{p y}$ & $m^{3}$ & 0.717 & $0.352 \sim 1.368$ \\
Lateral moment of inertia, $I_{z}$ & $m^{4}$ & 20.97 & $0.687 \sim 3.532$ \\
Lateral Plastic modulus, $S_{p z}$ & $m^{3}$ & 4.227 & $0.315 \sim 1.126$ \\
Cross sectional area, $A$ & $m^{2}$ & 0.556 & $0.212 \sim 0.549$ \\
Torsional moment of inertia, $J$ & $m^{4}$ & 1.317 & $0.681 \sim 2.792$ \\
\hline
\end{tabular}




\subsection{Influence of cable breakage durations and time-transient processes}

Depending on the causes, the actual cable failure processes could vary significantly from one to another. Some breakage may occur abruptly and the whole capacity of the cable gets lost within a very short time period, while others may partially lose some capacity until finally reaching total failure. As a relatively new topic, there is currently lack of sufficient information on defining the specific parameters

267 associated with various cable-loss scenarios from different causes, such as fire, lightning, blast and

268 vehicle impact. Although some parametric studies were conducted on several parameters of cable-loss 269 events (Ruiz-Teran and Aparicio 2009), realistic descriptions of cable-loss processes caused by different 270 incidents are not yet available. In the present study, general breakage phenomenon of stay cables is 271 assumed to occur independently from the causes. Based on the observations made in some existing 272 studies (Ruiz-Teran and Aparicio 2009; Mozos and Aparicio 2011), a cable loss process is defined in the 273 present study with two variables: total breakage time duration $t_{\text {total }}$ and time-transient cable area loss 274 function $l_{\text {rel }}$. Four total breakage time durations $t_{\text {total }}$ are assumed in the following sensitivity analyses: no 275 longer than 0.04 seconds, exactly 1.0 seconds, 2.5 seconds and 4.0 seconds. These time durations are 276 selected based on some reasonable assumptions developed in the limited existing studies. Since there is 277 little data or existing knowledge about the actual cable breakage process, the same assumption of 278 exponential function once adopted by Ruiz-Teran and Aparicio (2009) is followed here primarily because 279 of its computational convenience. The time-dependent cable area loss fucntion $l_{\text {rel }}$ is the ratio of lost cable 280 cross-section area to total cable cross-section area, which is assumed as an exponential function of the 281 relative breakage time $t_{r e l}$ for the latter three $t_{\text {total }}$ values. The time-dependent cable area loss fucntion $l_{\text {rel }}$ 282 can then be defined as:

$$
l_{r e l}\left(t_{r e l}\right)=t_{r e l}^{\alpha}
$$

284 where $t_{\text {rel }}$ is defined as the ratio of the elasped time $t_{\text {elap }}$ after breakage starts over the total breakage time 285 duration $t_{\text {total. }}$ a is the exponential factor, assumed to be $0.2,0.05,1,5$ and 20. 
By changing the exponential factor $\alpha$ as defined in Eq. (14), a breakage process can be modeled with

287 different patterns within the same breakage duration. For example, when $\alpha$ is set to be 1 , the function is a

288 linear breakage process indicating that the breakage occurs uniformly during the breakage duration. Since

289 the smallest $t_{\text {total }}$ is less than or equal to 0.04 seconds which is same as the time step used in the nonlinear

290 dynamic analysis, it is considered as a type of abrupt cable breakage scenario. The time-dependent cable

291 area loss function $l_{\text {rel }}$ for abrupt breakage scenario is defined as two similar cases: (1) the cable loses $100 \%$

292 area at the instant when the breakage starts $(t=0)$ (Process 1 as shown in Fig. 3), or (2) the cable remains

293 intact at the instant when the breakage starts $(t=0)$ and the cable loses $100 \%$ area at the beginning of

294 next time step $\left(t=t_{\text {total }}=0.04 \mathrm{~s}\right)$ (Process 7 as shown in Fig. 3).

295 Therefore, a total of 7 processes are defined: Processes 1 and 7 represent the two abrupt cable 296 breakage cases and Processes 2-6 represent the cases defined in Eq. (14) with the five $\alpha$ values as defined 297 above, respectively. The results of time-dependent cable area loss function $l_{r e l}$ versus $t_{r e l}$ are shown in Fig.

298 3. Considering the symmetric nature of cable loss processes as shown in Fig. 3, only Processes 1-4 are studied in the following sensitivity studies.

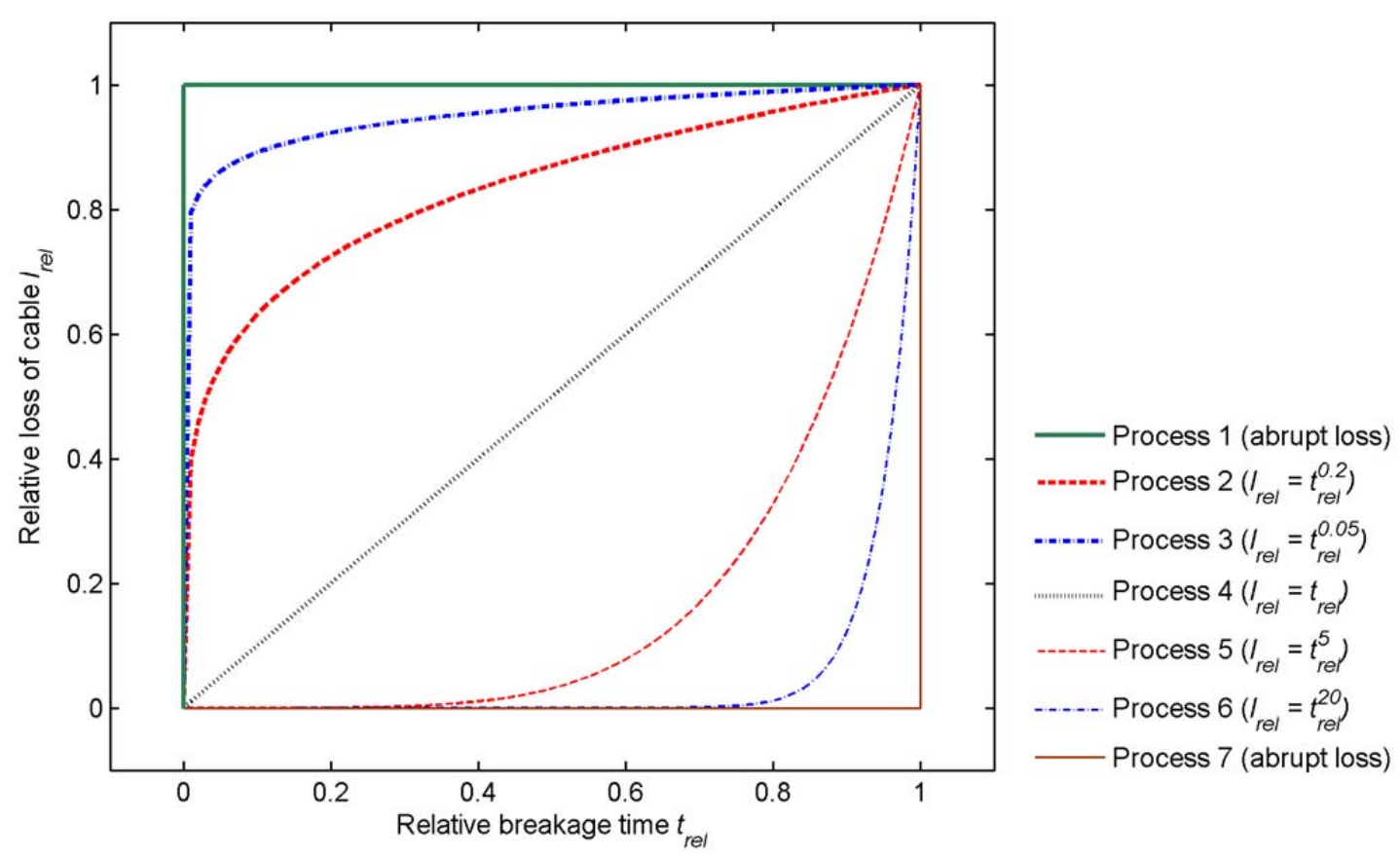


To better study the impact of cable loss duration and process without mixing contributions from other factors, no service load is considered and cable breakage starts at rest. To explore the influence from breakage duration, Cable 1a is assumed to break within four different total breakage time durations: abrupt (within 0.04 second), 1.0 second, 2.5 second and 4.0 second. For the abrupt breakage case (within $0.04 \mathrm{~s}$ ), the time-dependent cable area loss function $l_{\text {rel }}$ follows the Process 1 (i.e. $100 \%$ area lose at $t=0$ ). For the other three total breakage time durations (1.0, 2.5 and $4.0 \mathrm{~s}), l_{\text {rel }}$ follows Eq. (14) with a linear process ( $a=1$; Process 4). Assuming cable breakage starts at $t=0$, the time history responses of vertical displacement at the mid-span joint of the south bridge girder are shown in Fig. 4 for different total breakage time durations. It is clear that abrupt breakage will cause the largest dynamic response among 311 all the comparative cases with different total breakage durations. Larger dynamic response will be 312 induced in the breakage cases with shorter breakage durations especially when the breakage duration is 313 less than the fundamental period of the bridge $(2.5 \mathrm{~s})$. In the cases with breakage duration equal to or 314 larger than the fundamental period of the bridge, the dynamic response doesn't have remarkable change 315 when the total breakage duration increases.

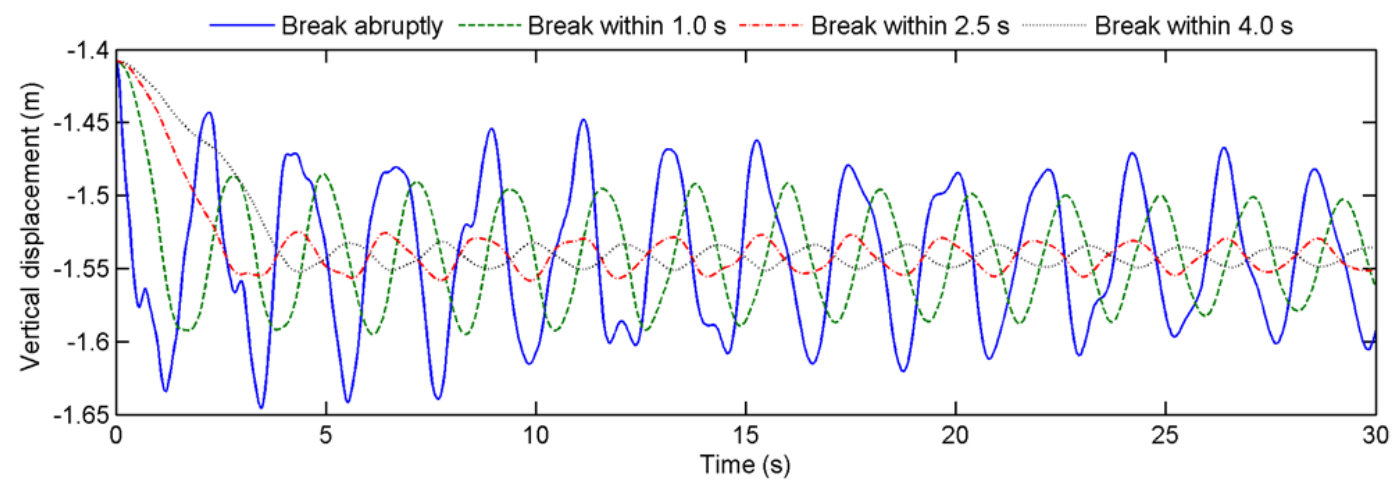

Fig. 4 Vertical dynamic responses at mid-span of south bridge girder with different breakage durations

319 In addition to total breakage time duration, the impact from time-dependent cable area loss function $320 l_{r e l}$ is also investigated. It is assumed that Cable 1a breaks within the total time duration of $1.0 \mathrm{~s}$ following 321 Processes 1 through 4 as defined previously. Assuming cable breakage starts at $t=0$, the vertical 
322 displacement time histories at the mid-span of the bridge girder are displayed in Fig. 5 for different 323 processes. It is found that Process 1 with abrupt loss of cable and Process 4 with linear loss of cable cause 324 the largest and smallest dynamic response among all four breakage processes, respectively. Compared to 325 the total breakage duration, the time-dependent cable area loss function $l_{\text {rel }}$ has relatively less impact on 326 the bridge response. The extreme dynamic responses induced in the cases with breakage processes 2 and 3273 are between the corresponding values in the case with breakage processes 1 and 4 . Dynamic response is 328 observed to become larger as the breakage process approaches abrupt loss process while it becomes 329 smaller as the breakage process approaches linear loss process. Since an accurate characterization of the 330 total breakage duration as well as the time-transient cable area loss function is not yet available, 331 uncertainties associated with these two factors are found necessary to be considered appropriately.

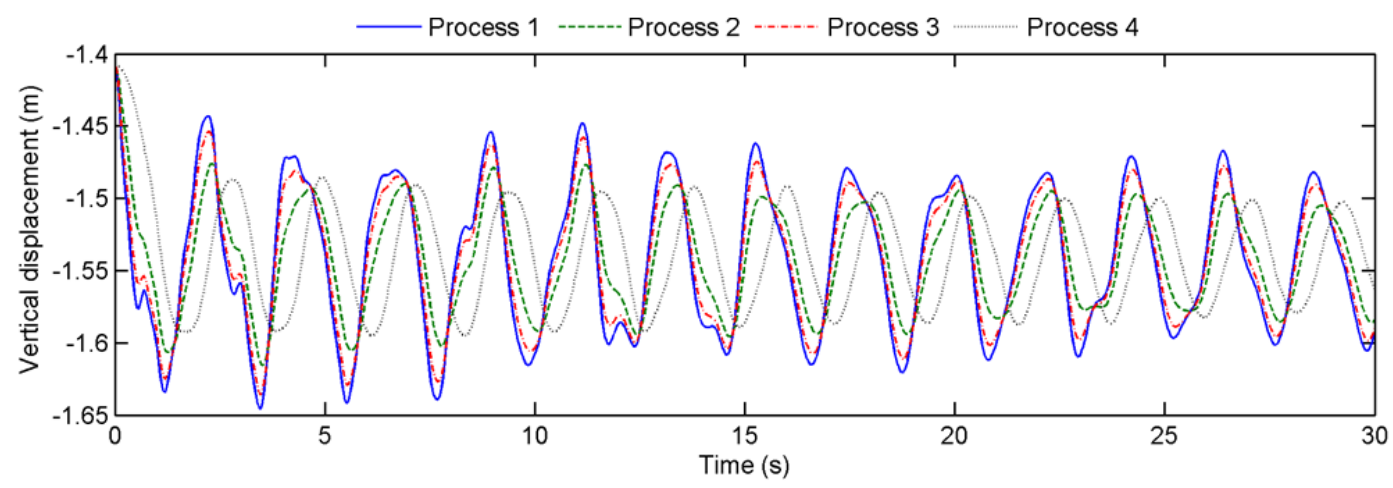

Fig. 5 Vertical dynamic response at mid-span of south bridge girder with different breakage processes In the parametric studies introduced above, abrupt breakage of cable is found to generally induce the largest dynamic response compared with other breakage processes, thus becoming the most critical breakage process of cable loss. The simulations in the following sections will be based on the abrupt cable loss assumption unless otherwise noted.

\subsection{Influence of the time instant when cable breakage occurs-dynamic initial states of} cable breakage

Long-span cable-stayed bridges vibrate considerably under service wind and traffic loads. Therefore, depending on the exact instant when cable breakage occurs, the initial state of cable breakage is not only 
dynamic with non-zero velocity and acceleration, but also exhibits stochastic characteristics. In a previous study by the authors (Zhou and Chen 2014a), dynamic initial states of cable breakage events only caused

344 by stochastic traffic were studied. In the present study, more comprehensive studies are conducted to 345 investigate the influence from the associated dynamic initial states of cable breakage subjected to both 346 traffic and wind service loads. The vertical and torsional displacements at the mid-span of the south 347 bridge girder are investigated assuming Cable 2 a fails abruptly at around 20 second when moderate traffic 348 and wind excitations of $20 \mathrm{~m} / \mathrm{s}$ are applied on the bridge.

349 Two dynamic extreme initial states for vertical displacement are selected at 19.32 second and 20.52 350 second, when the mid-span of the bridge girder moves to the uppermost and lowest positions, respectively. 351 For comparison purposes, another time instant, 20 second, is chosen between the two selected time 352 instants. Thus there are totally three representative dynamic initial states for vertical response, i.e. 353 breakage of Cable 2a starting at 19.32 second (dynamic initial state 1), 20 second (dynamic initial state 2) 354 and 20.52 second (dynamic initial state 3). Nonlinear dynamic analyses are conducted for the three 355 representative dynamic initial states with the vertical and torsional displacements at the mid-span joint of 356 bridge girder presented in Figs. $6 \mathrm{a}$ and $6 \mathrm{~b}$, respectively. It is found in Fig. $6 \mathrm{a}$ that the vertical 357 displacement response reaches the largest and the smallest extreme values with initial states 1 and 3 , 358 respectively. Similar to the observations made in the previous study (Zhou and Chen 2014a), the vertical 359 displacement of the bridge girder joint experiences local maximum and minimum values when the 360 breakage occurs at the time instant when the joint reaches the uppermost position and starts to move 361 downward, and the lowermost position and starts to move upward, respectively. As shown in Fig. 6b, the 362 maximum and minimum extreme initial states (i.e. initial state 1 and 3) identified based on vertical 363 response do not cause the maximum and minimum extreme response of torsional displacement response.

364 A comprehensive search among different breakage instants is made to identify the critical initial states for 365 torsional displacement. It is found that at the breakage instants of $20.08 \mathrm{~s}$ and $19.68 \mathrm{~s}$, the bridge torsional 366 displacement reaches the largest and smallest values with the presence of service loads, respectively. 
367

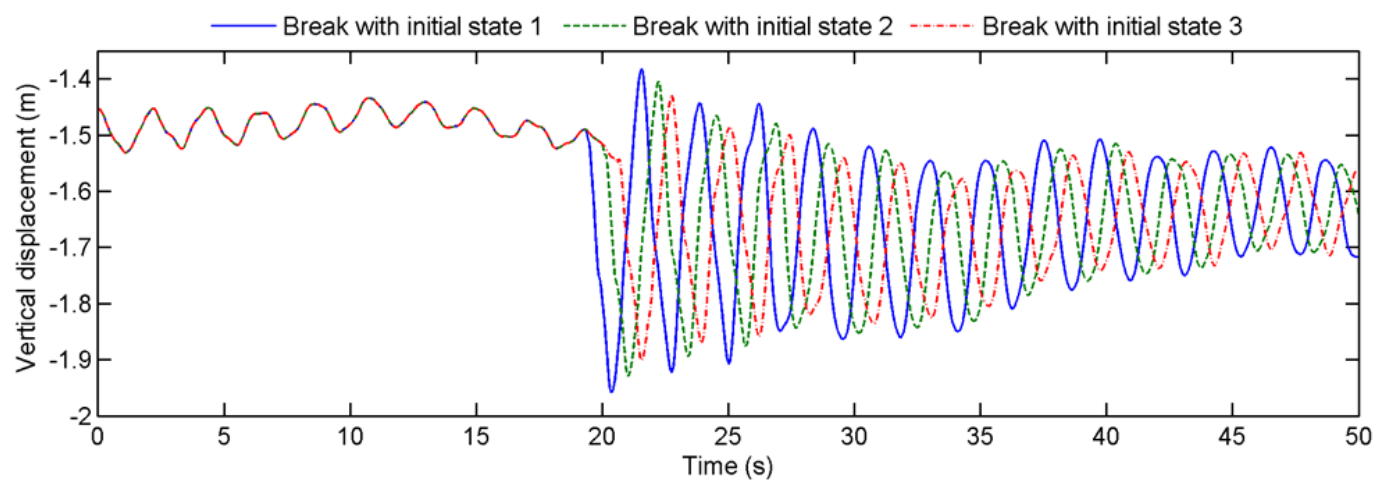

(a) Vertical displacement

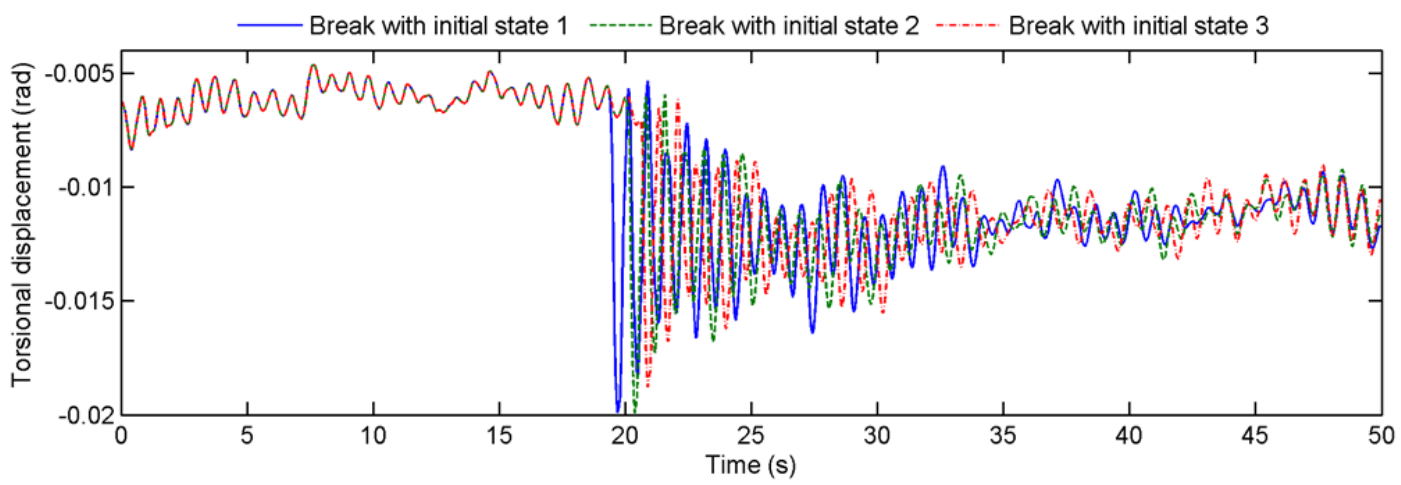

(b) Torsional displacement

Fig. 6 Dynamic displacement at mid-span of bridge girder with breakage initial states 1-3

Although critical initial states causing the maximum and minimum extreme responses in different directions (e.g., vertical and torsion) are not the same, some general trends can still be identified from the previous results. It is found that cable breakage will cause an instantaneous unbalance of the bridge system, accordingly exhibiting a sudden increase of the bridge response in particular directions before the bridge is able to rebalance. In the meantime, the bridge usually experiences dynamic vibrations under service loads when cable breakage occurs. The maximum response in any direction (e.g., vertical or torsional) will be achieved when the instantaneous bridge response under service loads reaches a local peak value, and starts to move in an opposite direction to the sudden movement direction of the bridge caused by the instantaneous unbalance as a result of cable loss. In other words, the instantaneous unbalance and rebalance process of the bridge structure subjected to cable breakage may reinforce or suppress the existing bridge vibration caused by the service loads, depending on the exact time instant the 
cable loss occurs. For various bridge components such as bridge girder, pylons and remaining cables, it is not realistic to determine universally unfavorable initial states of cable breakage events. Since the instant when cable loss occurs is nearly impossible to predict, the bridge response exhibits certain randomness with different breakage dynamic initial states in a cable breakage event, which require appropriate 387 probabilistic simulation based on the reliability theory.

\subsection{Impact on internal forces from cable loss events - A baseline scenario}

It is possible for cable breakage events to start at rest without the presence of excitations from stochastic traffic or turbulent wind, especially when sufficient warning time is available to evacuate the traffic and wind is very mild at the same time. Such a scenario also serves as a baseline case, which

392 provides useful information about the impact on bridge performance from cable breakage only, without 393 mixing contributions from service loads. Cable loss can occur on any cable of the bridge and a 394 comparative study of single-cable loss event occurring on three representative cables is carried out. 395 Single-cable breakage scenario of cable 1a, 2a or 3a is assumed to occur respectively at 20 second to be consistent with the following studies. However, it is noted that cable breakage in the baseline scenario starts at rest and no dynamic excitations are applied during the whole simulation time period. The impact on the dynamic internal forces of the bridge girder, pylon column and cables is investigated through conducting nonlinear dynamic analyses on the three cable breakage scenarios.

\subsubsection{Dynamic response on bridge girder}

401 The extreme dynamic responses in terms of vertical bending moment on the bridge girder are 402 obtained from the nonlinear dynamic analysis of the bridge for breakage cases of Cable 1-3a. For 403 comparison purposes, the corresponding static bridge response of the intact bridge without cable loss is 404 obtained from nonlinear static analysis of the bridge. The vertical bending moments along the whole 405 bridge under all the three cable loss events and one undamaged bridge case are plotted in Figs. 7a-b. 406 Positive and negative vertical bending moments on the bridge girder are defined as those causing tension 407 on the bottom slab and the top slab of the bridge girder, respectively. It is found from Fig. 7a that cable 
breakage will generally cause the largest increase of positive bending moments at the girder segments

409 closest to the failed cable. Among all the three cases, breakage of Cable 1a causes the largest extreme

410 positive bending moments at the middle point of the main span, which may control the bridge girder

411 design against single-cable breakage event. For all the three representative cable-breakage scenarios,

412 positive dynamic bending moments at the corresponding bridge girder locations closest to the failed

413 cables all exhibit considerable amplification from those at the same locations when the bridge was intact.

414 Among the three representative cable-breakage events, the largest dynamic amplification is observed in

415 the breakage case of Cable 2a. Different from positive bending moments, as shown in Fig. 7b, extreme

416 negative bending moments on the bridge girder closest to the failed cable do not exhibit significant

417 amplifications from those of the intact bridge, rather causing significant increase on the bridge girder

418 locations around the nearest remaining cables. For example, breakage of Cable 1a will cause notably

419 amplified negative bending moment at the girder locations connecting Cable 2a and 3a. Similarly,

420 breakage of Cable 2a will considerably amplify the negative bending moments at the girder locations

421 connecting Cable 3a and 1a. The maximum dynamic response ratios (DRR), defined as the ratio of the

422 response in the breakage case to that in the undamaged case, are -4.17 and 1.24 for vertical bending

423 moments at the girder location connecting Cable 2a and mid-span, respectively. The negative sign of the

424 DRR indicates the change of moment direction. It should be noted that the absolute extreme bending 425 moment at the mid-span is still larger than that at the girder location of cable $2 \mathrm{a}$ in the above case 426 although the DRR of the latter is larger.

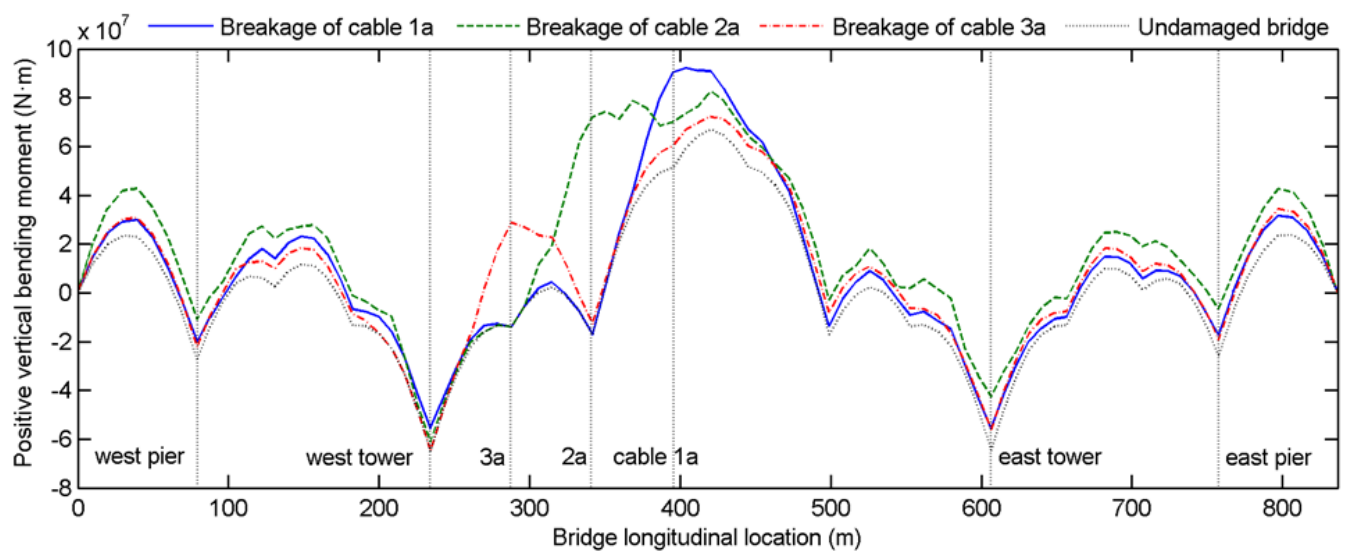




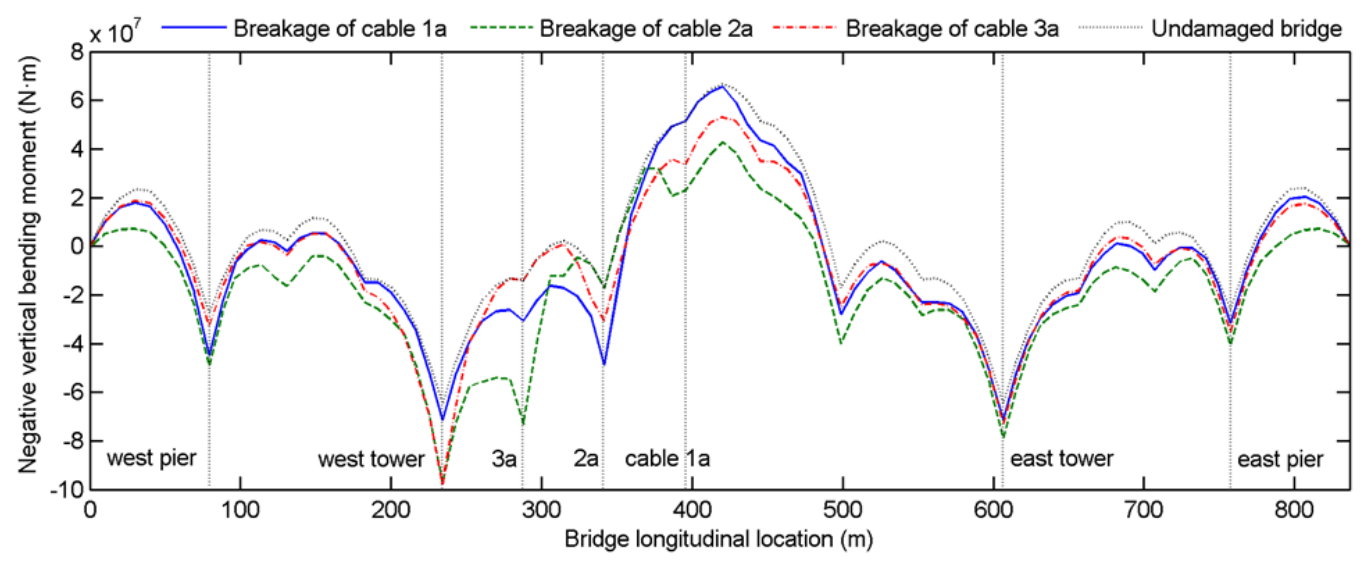

(b) Negative vertical bending moments

Fig. 7 Extreme vertical bending moments along bridge girder

434 larger torsional moments. The nonlinear time history analyses provide the information about the extreme 435 positive and negative torsional moments along the bridge girder as shown in Figs. 8a and b, respectively.

436 The positive torsional moment is defined following the right hand rule rotating about the positive $x$ 437 direction. It is seen from Figs. 8a-b that positive and negative extreme torsional moments are generally 438 anti-symmetric about the girder location closest to the cable breakage. For both positive and negative 439 torsional moments, significant increase has been observed at the girder locations near the failed cable and 440 also on nearby cables. Among all the three representative cable loss events, the breakage of Cable $2 \mathrm{a}$ 441 generally induces the largest extreme torsional moment and also dynamic amplifications as compared to 442 the undamaged case. The maximum DRRs in the breakage case of Cable 2a for torsional moment are 44311.95 and -24.47 at the girder location connecting Cable $2 \mathrm{a}$ and mid-span, respectively. This result shows 444 that some individual cable breakage events could be more critical than others in terms of internal forces 445 on the bridge, and the critical cable-breakage scenario, possibly controlling the design, needs to be 446 appropriately identified on a case-by-case basis. 


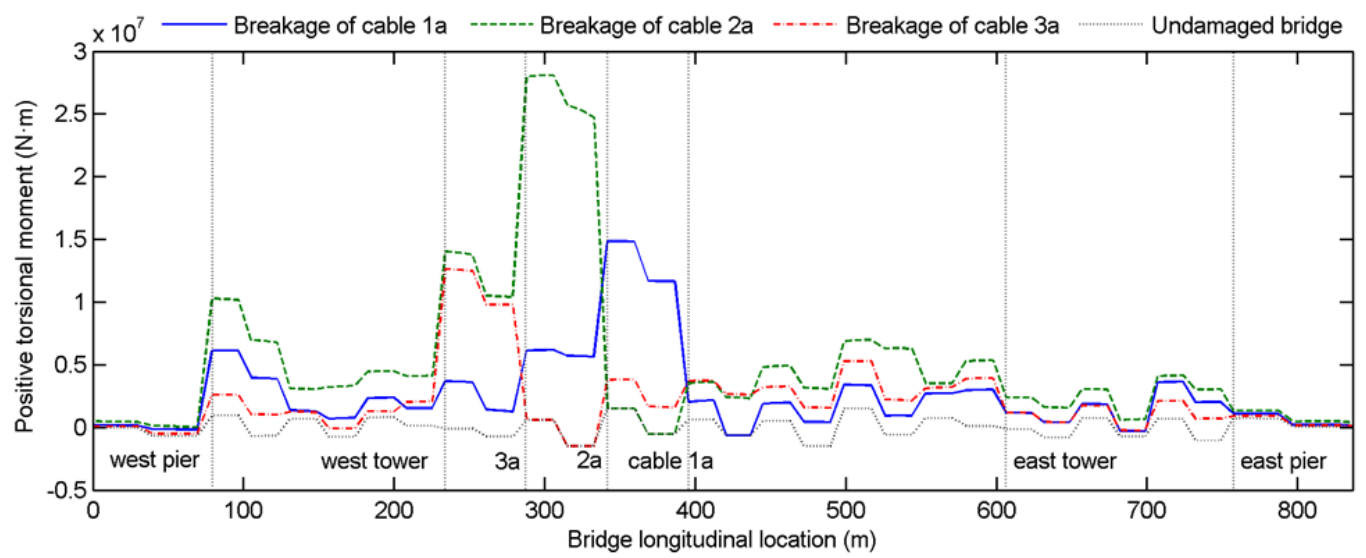

(a) Positive torsional moments

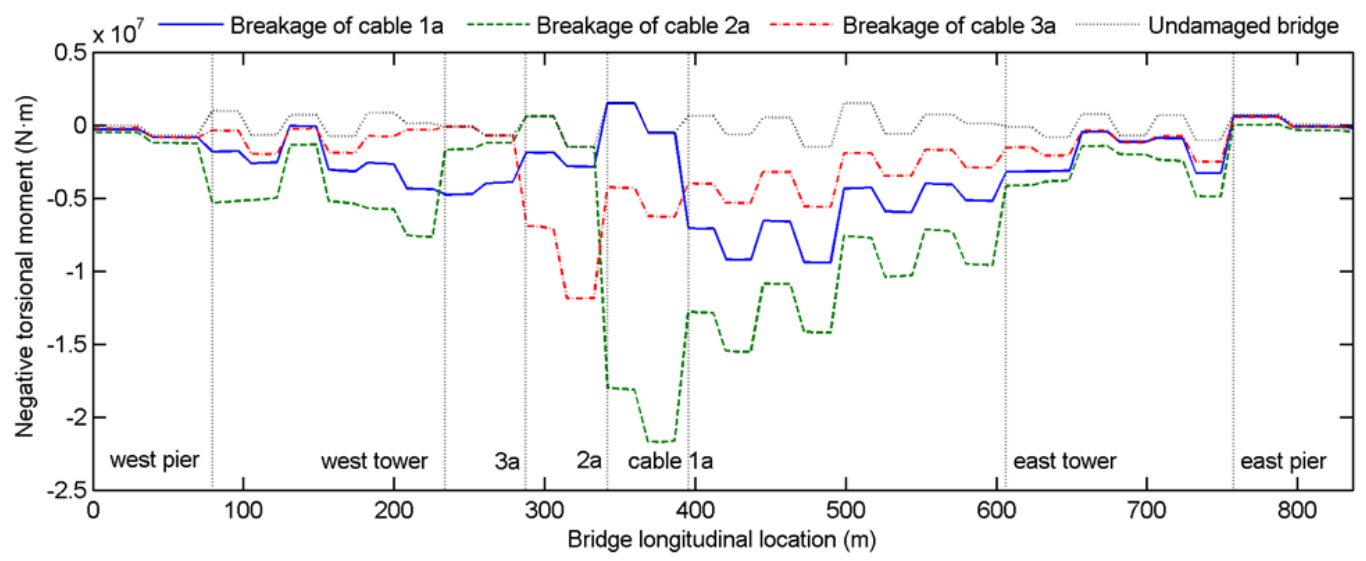

(b) Negative torsional moments

Fig. 8 Extreme torsional moments along bridge girder

\subsubsection{Dynamic response on bridge pylons}

453 Extreme positive and negative longitudinal bending moments of the south column on the west pylon

454 are evaluated and compared with those on the same pylon column of the intact bridge from nonlinear

455 static analysis (Fig. 9). Positive longitudinal bending moments of the bridge pylon are defined as those 456 causing tension on the east side and compression on the west side of the pylon column. 

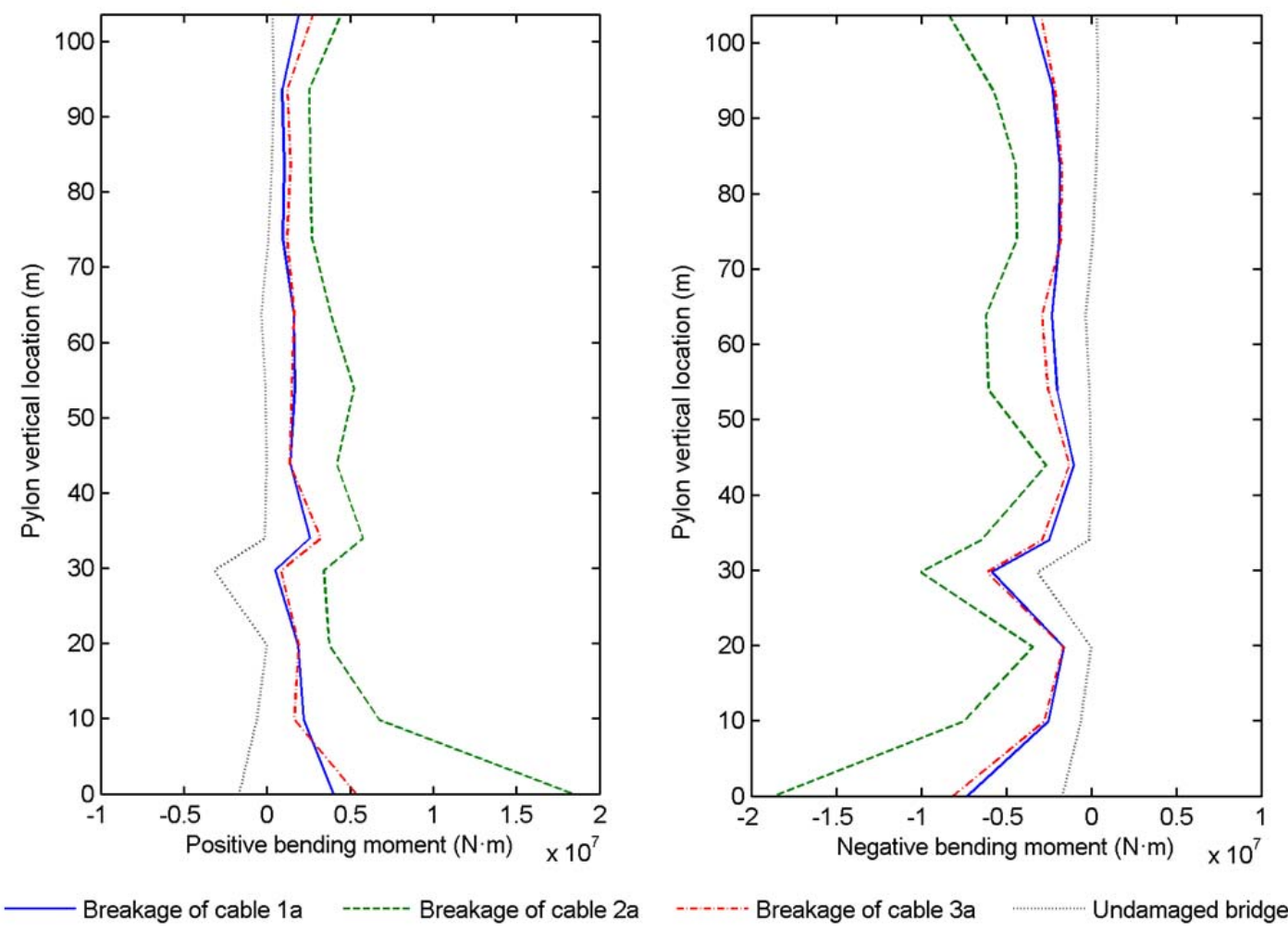

Fig. 9 Extreme longitudinal bending moments along bridge pylon increase of extreme bending moments on the pylon column in both positive and negative directions,

461 particularly at the base of the pylon column. Compared to Cable 1a and Cable 3a, breakage of Cable 2a 462 induces notably larger extreme bending moments on the pylon column in both positive and negative 463 directions. It is found that the critical location for the pylon column subjected to cable breakage events is 464 the pylon base with the largest positive and negative longitudinal bending moments. As compared to the 465 undamaged case, the DRRs at the pylon base can reach up to -10.9 and 11.0 for positive and negative 466 bending moments, respectively.

467 The extreme torsional moments along the bridge pylon in positive and negative directions are shown 468 in Fig. 10. It is again found that breakage of Cable 2a induces the largest torsional moments along the 469 pylon column among the three breakage cases. The critical location in terms of torsional moments on the 470 pylon column for the three cable breakage cases is at the pylon base and the DRRs for torsional moment 
472 pylon column.
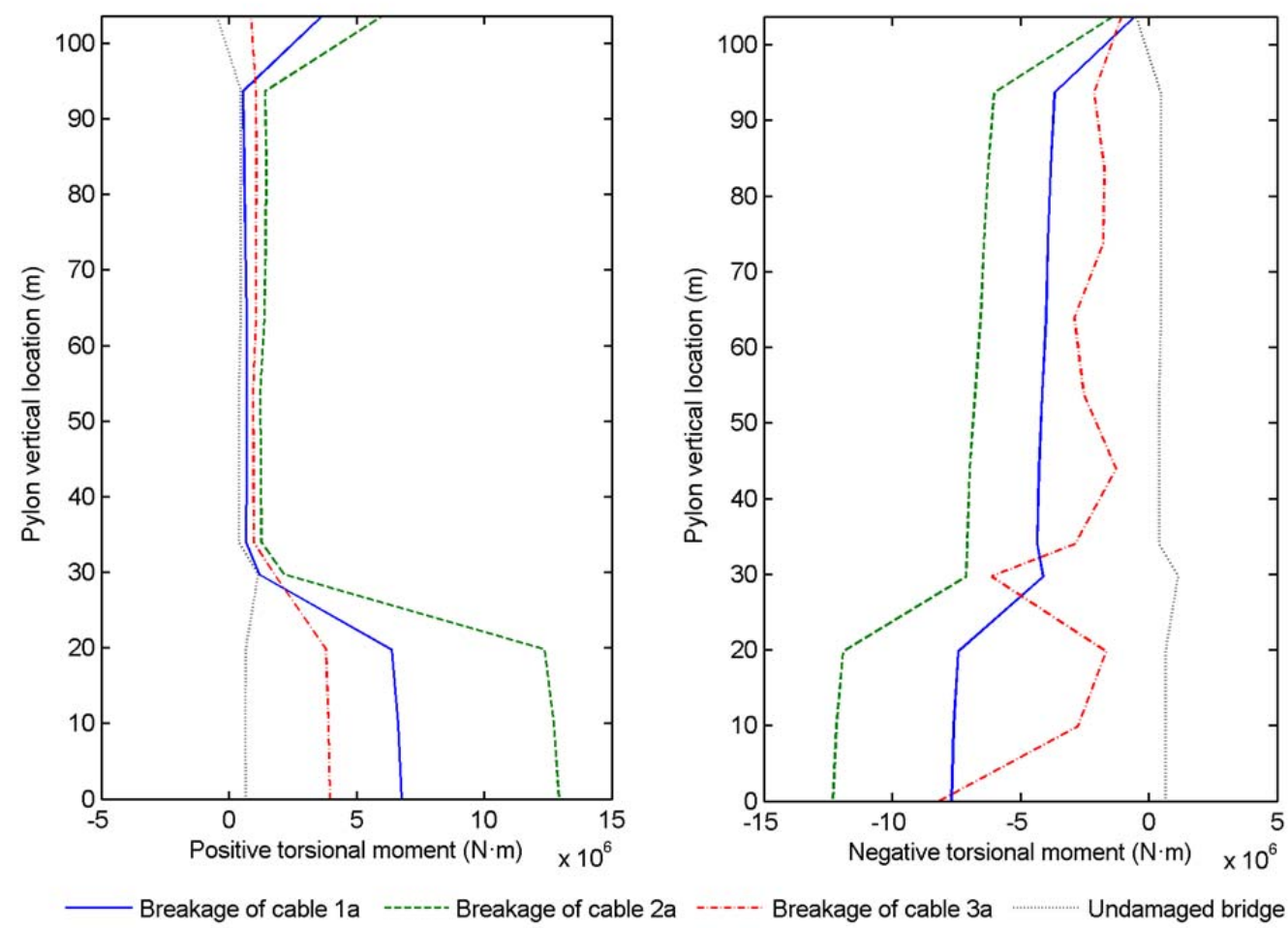

Fig. 10 Extreme torsional moments along the west bridge pylon

\subsubsection{Dynamic response on bridge cables}

The dynamic responses of the remaining cables on the bridge are investigated in the three single-cable the static cable forces of the undamaged bridge structure (Fig. 11). Not surprisingly, it is found that the

480 remaining cables close to the ruptured cable are more likely to be influenced by a cable breakage incident.

481 For instance, abrupt breakage of Cable 1a induces the largest increase of tension force at Cable 2a from 482 that of the undamaged bridge. The DRRs at the locations of Cables 1a and $3 \mathrm{a}$ in the breakage case of 483 Cable 2a are 1.77 and 2.76, respectively. Although it seems significant that the remaining cable forces 484 will increase by $77 \%$ to $176 \%$ subjected to a single-cable loss event compared with those on the 485 undamaged bridge, the local response amplification on the bridge girders and pylons as discussed above 
are actually much more substantial in terms of DRR. It is found that the breakage of Cable 2a generally

487 induces the largest extreme tension forces on the remaining cables among the breakage cases of Cable 1-

488 3a. Similar conclusions are drawn for the internal force or moment response of the bridge girder and

489 pylon. The influence of the cable breakage event on the bridge response depends on several factors, such

490 as the stay cable being on the side span or main span, the distance between two adjacent remaining cables,

491 the cable inclination angle and the cable length, etc. In the present study, the distances between any two

492 adjacent remaining cable/pylon supports for the Cable 1a, 2a and 3a are very close, which are $103.5 \mathrm{~m}$,

$493107.6 \mathrm{~m}, 107.6 \mathrm{~m}$, respectively. Therefore, the distance between two adjacent remaining cable/pylon

494 supports is not the main contributing factor for the different bridge responses. The fact that breakage of

495 Cable 2a causes largest dynamic responses of the bridge is probably because the ability for the east bridge

496 tower (not connecting with the breaking cable) to withhold additional girder gravity force after cable

497 breakage occurs outweighs the force effects on the west bridge tower (connecting with the breaking cable)

498 due to the larger inclination angle of the breaking cable. It is noted that the most disadvantageous

499 breakage situation may vary among different bridges and the accurate determination of the most

500 disadvantageous scenario requires finite element analysis of the breakage events on the specific bridge.

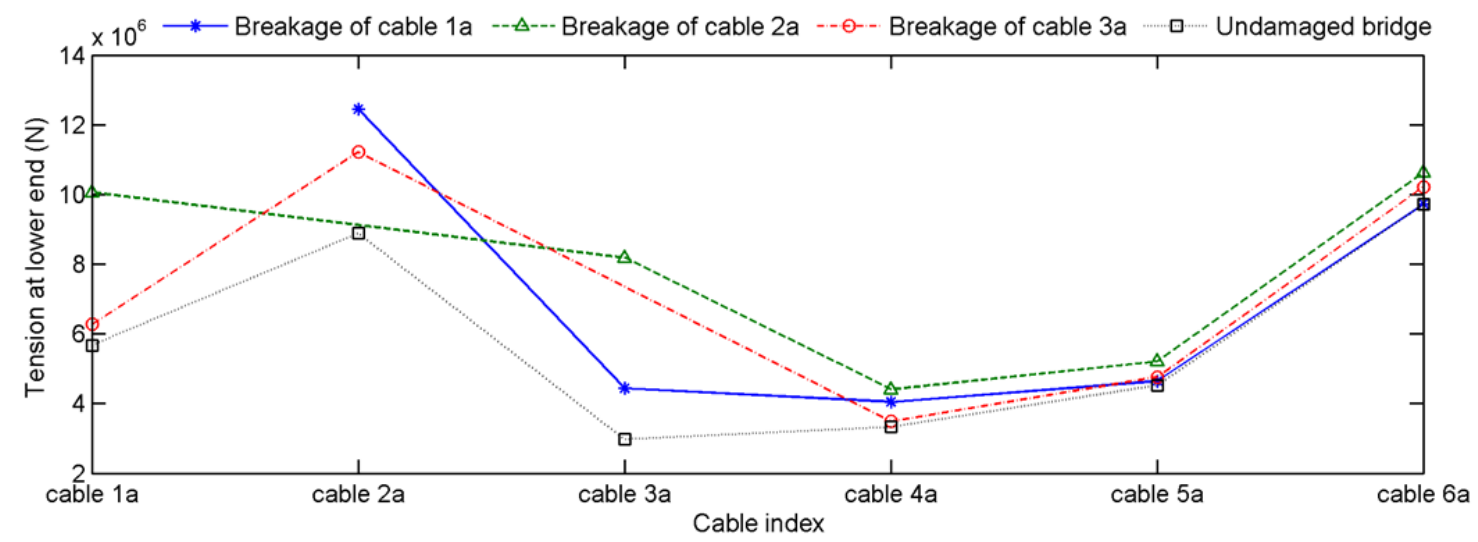

Fig. 11 Maximum tension forces of remaining cables in the breakage case of cable 1-3a 


\subsection{Impact on internal forces/moments due to excitations from stochastic traffic and} wind

\subsubsection{Dynamic excitations from stochastic traffic}

The panic driving behavior of the vehicles may exist after the drivers are able to detect the incidents causing the cable loss. The influences of the emergency incidents on the driving behavior are very complicated and unfortunately existing related studies are still very limited. Due to the lack of appropriate

509 model and data to characterize the panic driving behavior, the driving behavior following the cable

510 breakage is assumed to remain unchanged in the present study. Such an assumption is valid for the

511 instants immediately following the cable-loss incidents before the drivers are able to detect and respond to

512 the incidents and may be approximate for the extended time after the cable-loss incidents. It is found in 513 previous sections that breakage of Cable 2a will generally cause the largest dynamic internal forces or 514 moments on bridge girder, pylon and cables among the three breakage cases of Cable 1a, 2a and 3a. In 515 this section, the bridge dynamic performance is investigated for the cable breakage case of Cable 2a with 516 the presence of stochastic traffic. As discussed earlier, stochastic traffic flow is generated using the CA 517 model with two different traffic densities, which are 31 and 16 vehicles per kilometer per lane 518 representing busy traffic and moderate traffic conditions, respectively. The breakage of Cable $2 \mathrm{a}$ is 519 assumed to occur at 20 second in the three comparative cases with busy traffic, moderate traffic and no 520 traffic, respectively. In previous sections, the critical locations of study have been identified at several 521 locations when Cable 2a is assumed to fail, such as mid-span joint of the south bridge girder for vertical 522 bending moment, the bridge pylon base for longitudinal bending moment, and Cable 1a for tension force. 523 The dynamic responses of these corresponding critical locations will be studied respectively in the 524 following to evaluate the critical impacts from excitations due to different service loads.

525 The time histories of vertical bending moment at the mid-span of the bridge south girder, longitudinal 526 bending moment at the pylon base and tension force of Cable 1a are demonstrated in Figs. 12a, b and c, 527 respectively. Before the cable breakage occurs at 20 second, the pre-breakage internal forces or bending 528 moments of the bridge girder or cables are generally larger in the case with busy traffic than those in the 
case with moderate traffic or no traffic. In contrast, the pre-breakage moment at the base of the bridge

530 pylon is small and exhibits little difference under different traffic densities. As shown in Fig. 12, for the

531 three bridge members (i.e. bridge girder, pylon and Cable 1a), post-breakage (after $t=20 \mathrm{~s}$ ) responses all

532 show significant amplification over the respective pre-breakage responses. The largest extreme values of

533 post-breakage responses for all the three bridge members actually occur during the first 1-2 cycles, rather

534 than immediately after cable breakage occurs. For bridge girder (Fig. 12a) and Cable 1a (Fig. 12c),

535 different traffic densities do cause considerably different extreme post-breakage responses and

536 interestingly, moderate traffic condition, instead of busy traffic condition as expected intuitively, causes

537 the largest post-breakage extreme response at mid-span of bridge girder. This phenomenon may be caused

538 by several possible reasons. Considering that the traffic flow is stochastically simulated using CA model,

539 two different patterns of traffic flow may have different extreme response of the bridge in a cable

540 breakage event even for the same traffic flow density because of the stochastic nature of the simulated

541 traffic flow. The different dynamic excitations from traffic loads may affect the breakage initial state and

542 further influence the extreme response after cable breakage occurs. In addition, due to the fewer vehicles

543 in the moderate traffic flow than in the busy traffic flow, the vehicles in the moderate traffic flow

544 generally move faster than those in the busy traffic flow, which may generate larger interaction effects

545 between the bridge and vehicles. For the bridge pylon (Fig. 12b), traffic has little effect on the post-

546 breakage base moment, similar to the observation of pre-breakage response. Unlike the bending moment

547 on bridge girder and tension force of Cable 1a, cable breakage itself causes large cyclic change of the

548 bending moment at pylon base with close-to-zero mean value.

549

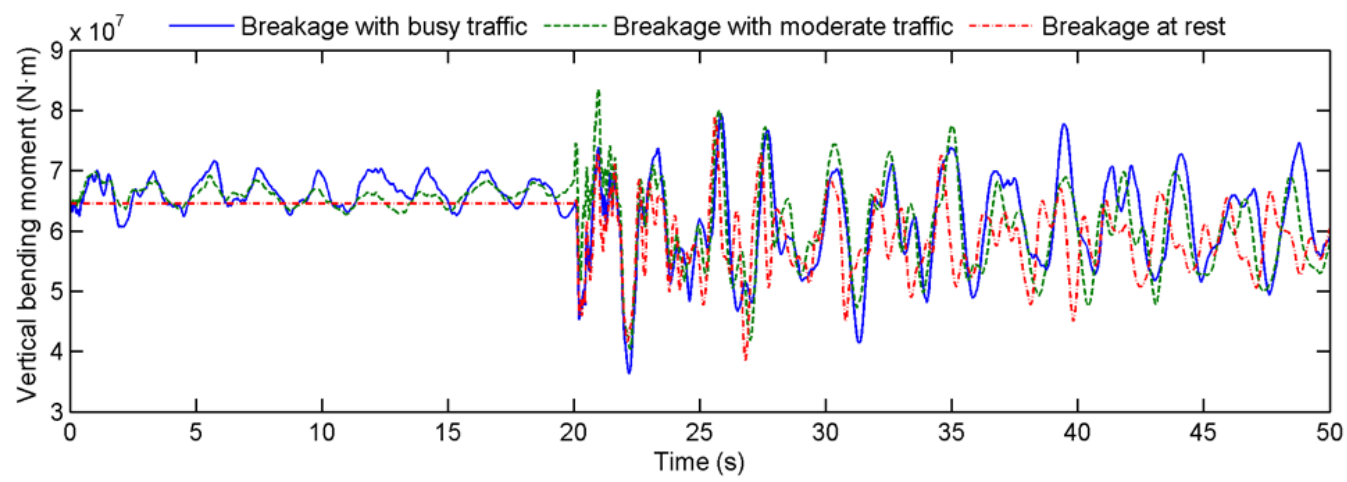




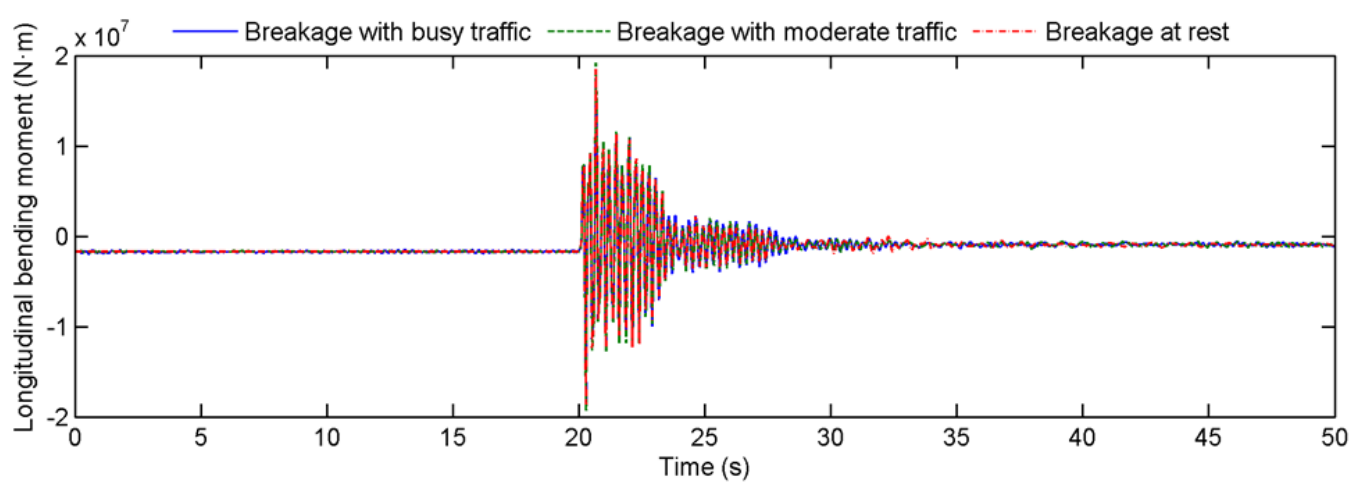

(b) At base of bridge pylon column

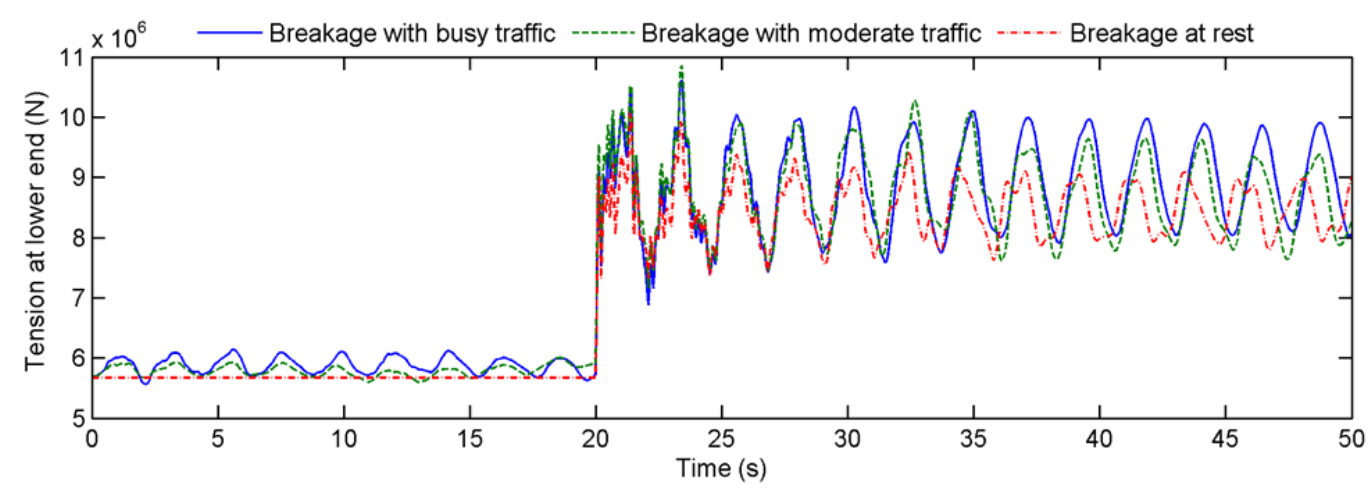

(c) At cable 1a

Fig. 12 Internal force/moment histories at critical location of bridge girder, pylon and cables

\subsubsection{Dynamic excitations from wind}

In this section, the dynamic performance of the prototype bridge subjected to single-cable breakage

(Cable 2a) is evaluated when only wind excitation is considered. Two steady-state wind speeds are studied in the cable breakage cases of Cable 2a starting at 20 second: $20 \mathrm{~m} / \mathrm{s}$ representing moderate wind and $40 \mathrm{~m} / \mathrm{s}$ representing strong wind in order to investigate the influence on dynamic response of the

562 wind scenario when the bridge is typically closed to traffic. Under $20 \mathrm{~m} / \mathrm{s}$ wind speed, traffic may still be

563 remained on the bridge. The wind-only case with $20 \mathrm{~m} / \mathrm{s}$ wind speed in this section is therefore primarily

564 for investigation and comparison purposes. The time histories of torsional moment at the mid-span of the 

Figs. 13a, b and c, respectively.

567

568

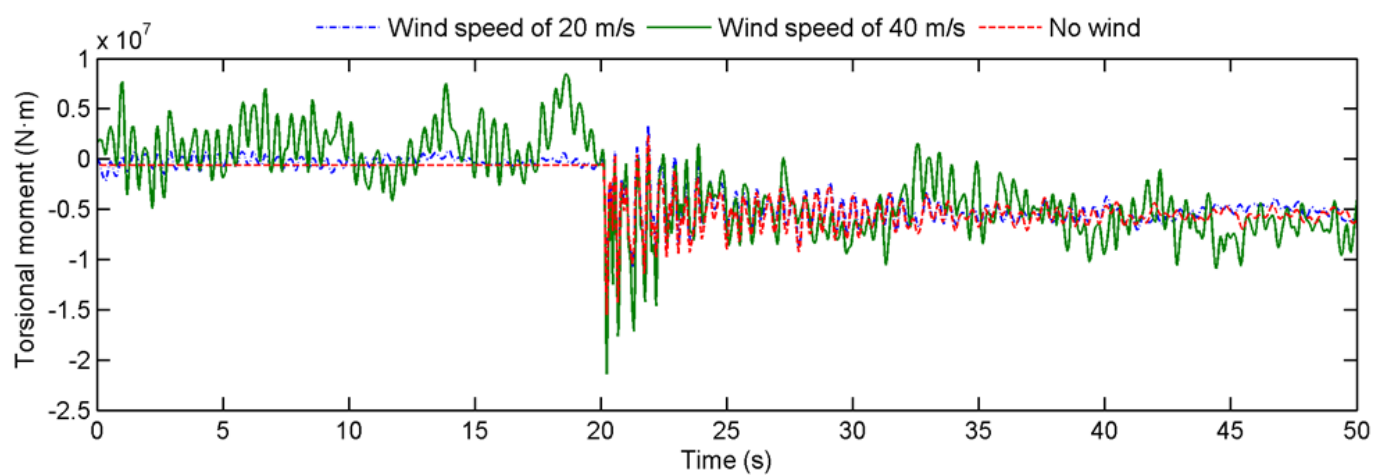

(a) Torsional moment at mid-span of south bridge girder

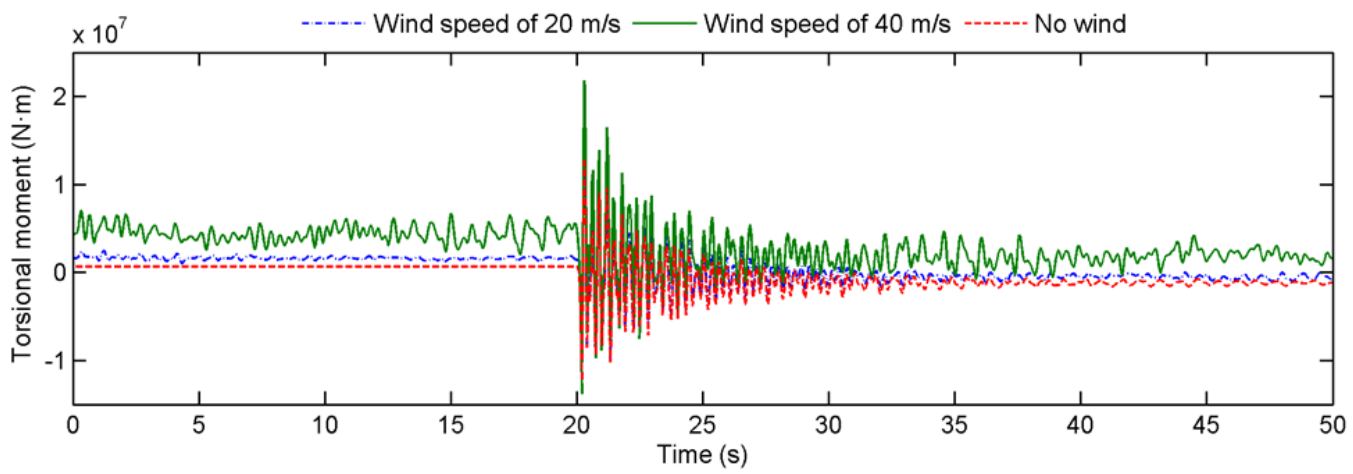

(b) Torsional moment at pylon base

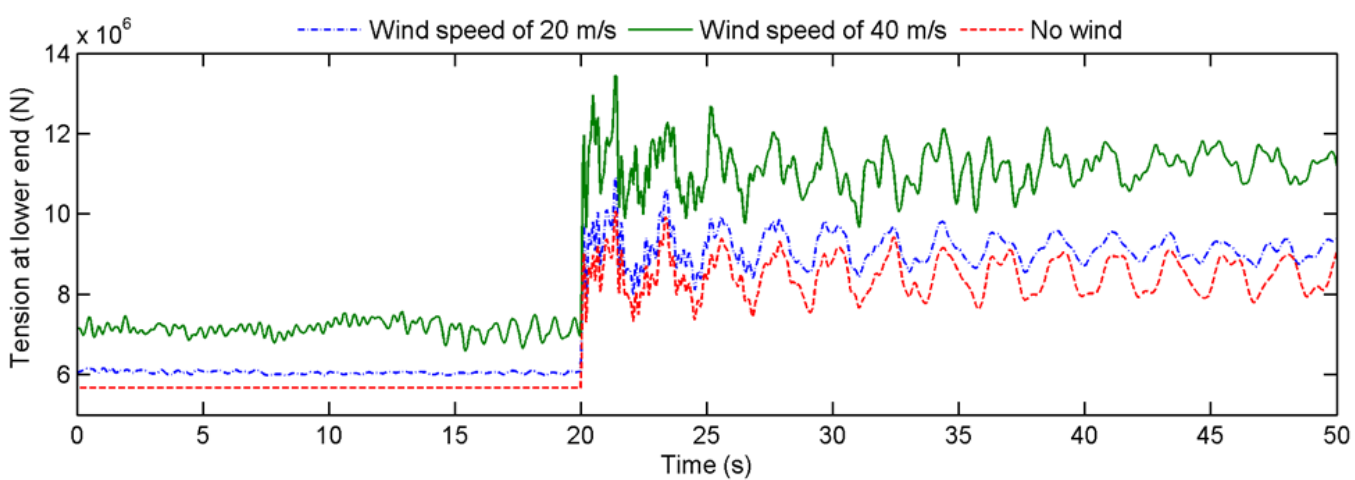

(c) Tension force of cable 1a

Fig. 13 Internal force/moment of bridge in the breakage cases of Cable 2a

It is found that as wind speed increases, the initial static displacement of the bridge from nonlinear static analysis under steady-state wind load and gravity increases accordingly. Different from the traffic- 
only cases, the torsional moments of the three bridge components, including bridge pylon, all exhibit

577 increased extreme values for pre-breakage and post-breakage responses when wind speed increases.

578 Similar to traffic-only case in the previous section, cable breakage causes dramatic increase of the 579 torsional moments on the bridge girder and pylon, as well as tension forces for the cable. Because of the

580 new equilibrium position of the bridge, post-breakage dynamic responses of the three bridge members all 581 maintain different mean values from the respective ones for the pre-breakage responses. Except for bridge 582 cables, of which the tension force reaches the maximum extreme values 1-2 cycles after the occurrence of 583 cable breakage, torsional moments for both bridge girder and pylon base reach the peak values during the 584 first cycle after the occurrence of cable breakage. In addition, it is observed that the dynamic impact from 585 cable breakage increases nonlinearly as wind speed increases. This is mainly due to the fact that the wind 586 forces, including static, self-excited and buffeting forces are closely related to the square of the steady587 state wind speed. The demands for internal forces or moments of the bridge structure increase more 588 significantly as the wind speed increase from $20 \mathrm{~m} / \mathrm{s}$ to $40 \mathrm{~m} / \mathrm{s}$ than those with wind speed increasing 589 from 0 to $20 \mathrm{~m} / \mathrm{s}$.

\subsubsection{Combined dynamic excitations from stochastic traffic and wind}

591 Although a consistent criterion is not yet available across the United States and the rest of the world, 592 some bridges are often closed to traffic for safety concern when mean wind speed at bridge girder gets 593 high (Chen et al. 2009). For example, AASHTO assumes a bridge may be closed to traffic when wind 594 speed exceeds $25 \mathrm{~m} / \mathrm{s}$, although under which many bridges are actually not closed (Chen and Wu 2010). 595 To simulate a typical service condition with both normal traffic and relatively strong wind, moderate 596 traffic and wind loads with a steady-state wind speed of $20 \mathrm{~m} / \mathrm{s}$ are applied on the prototype bridge. The 597 combined effects of stochastic traffic and wind load are investigated assuming Cable 2a fails at 20 second 598 in the three comparative cable-breakage cases: with stochastic traffic and wind, with only stochastic 599 traffic and with only wind. The time histories of vertical bending and torsional moments at the mid-span 600 joint of the south bridge girder are shown in Figs. 14a and 14b, respectively. It is found that the extreme 601 dynamic response of the bridge after cable breakage generally reaches the largest values when both 
stochastic traffic and wind are applied. Before cable-breakage occurs, torsional moment of the bridge is dominated by wind excitations while vertical bending moment is influenced by both wind and traffic excitations. The post-breakage dynamic responses in the case with both traffic and wind and the case with only stochastic traffic are compared. It is found that the inclusion of wind excitation on top of the stochastic traffic excitation causes slight increase of the peak moments immediately after cable breakage,

607 but gradually suppresses the dynamic responses in both vertical and torsional directions, evidenced by 608 reduced standard deviation. In addition, it is demonstrated that the dynamic impact due to cable breakage 609 damps out faster when wind excitations are included, likely due to the aerodynamic damping effects 610 through bridge-wind coupling. Similar observations are made by comparing the bending/torsional 611 moments at the base of the pylon column and tension forces at Cable 1a among the breakage cases with 612 different combinations of wind and traffic. It is found that the extreme post-breakage response in terms of 613 vertical bending moment in the wind-only case has considerably smaller value than those in the other two 614 cases. Such a phenomenon suggests that although wind load usually dominates torsional moment, 615 stochastic traffic load is essential to capturing the extreme post-breakage response of the bridge girder in 616 terms of both bending and torsional moments. It can be concluded from the comparative studies that fully 617 coupled consideration of the bridge-wind-stochastic traffic is important to cable-breakage simulation of 618 long-span bridges by capturing the extreme values of both pre-breakage and post-breakage responses.

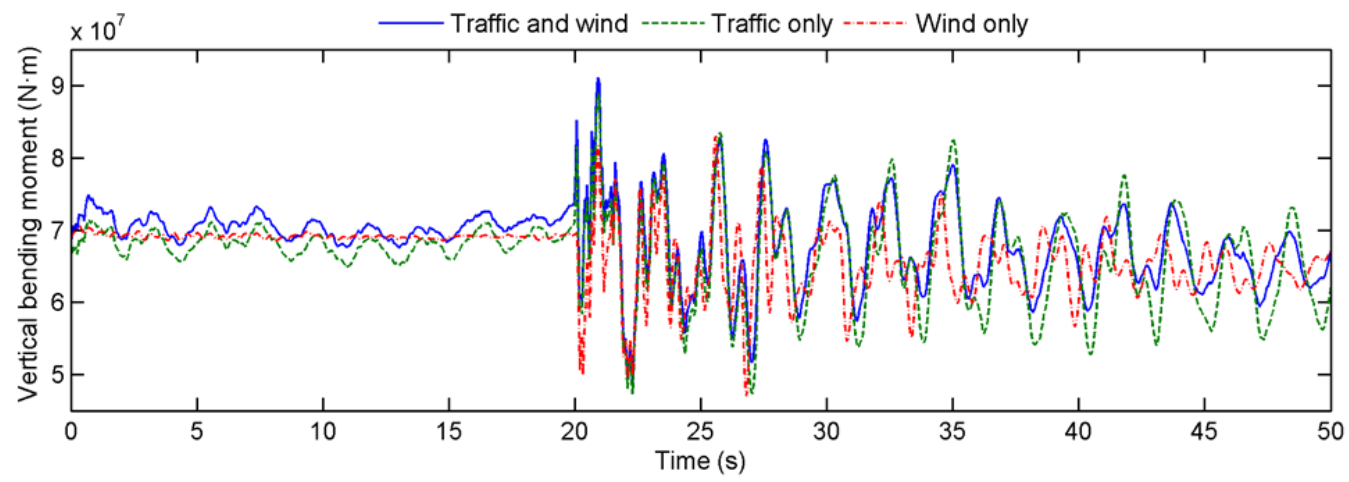

(a) Vertical bending moment 


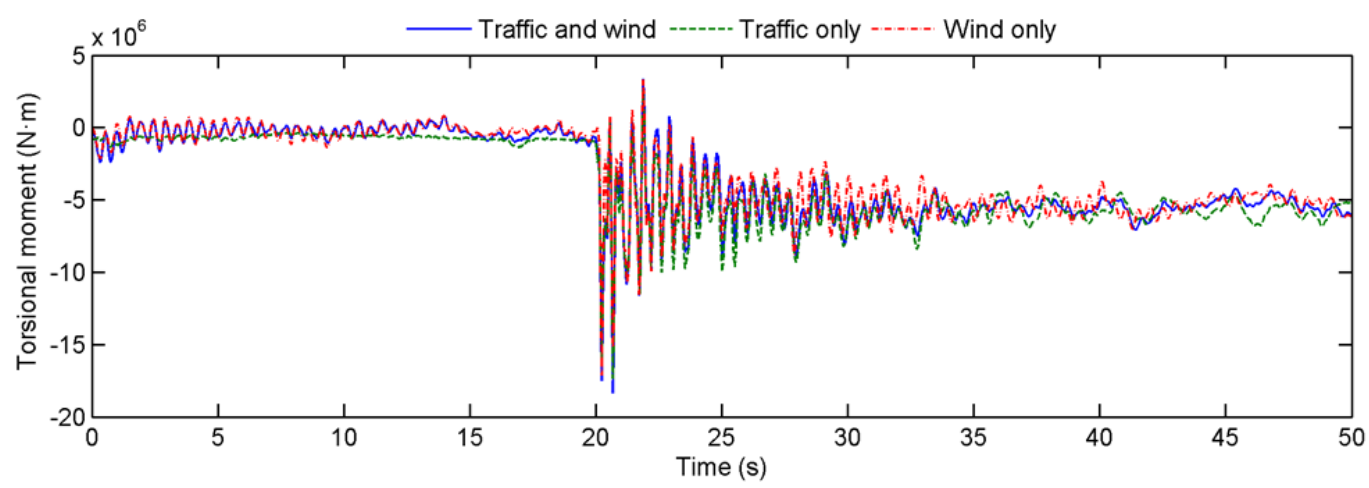

(b) Torsional moment

Fig. 14 Vertical bending and torsional moment in the breakage cases with traffic and/or wind

\section{Response Envelope Analysis and Comparison with the Design Specification}

Without specifying the applicable types and spans of cable-stayed bridges, PTI regulations (PTI 2007) are usually followed to carry out the design, testing and installation of stay cables, in which the equivalent nonlinear static analysis approach is recommended. Due to the unique characteristics such as sensitivity to wind and the large amount of traffic for long-span cable-stayed bridges as compared to those with shorter spans, the applicability of the recommended analytical approach on long-span cable-stayed bridges is not clear. In the following section, a comparison between the results from the proposed nonlinear dynamic analysis approach and those from the approximated approach recommended by the PTI design specification is made. From the design perspective, response envelopes along major bridge elements are crucial in order to capture the critical sections and also the worst-case scenarios. Single cable breakage events with the presence of both stochastic traffic and wind excitations at a steady-state wind speed of 20 $\mathrm{m} / \mathrm{s}$ are selected for the following study. For comparison purposes, the equivalent nonlinear static analysis with a DAF (Dynamic Amplification Factor) of 2.0 as recommended by PTI (2007) is also conducted on the prototype bridge. The proposed nonlinear dynamic analysis approach adopts stochastic traffic load, which cannot directly be applied to the equivalent nonlinear static analysis. Existing design traffic load in the AASHTO LRFD specification (AASHTO 2010), such as HL-93 primarily calibrated from and used for short-span bridges, is not directly applicable to long-span bridges. In order to make rational 
comparison, an equivalent uniformly distributed traffic load $3.37 \mathrm{kN} / \mathrm{m}$ along the bridge is quantified,

642 under which the static vertical displacement at the midpoint of the main span equals the mean value of the

643 dynamic vertical displacement of the proposed nonlinear dynamic analysis with stochastic traffic flow. To

644 consider the wind loads on bridges, the steady-state wind forces corresponding to wind speed of $20 \mathrm{~m} / \mathrm{s}$

645 are also applied on the bridge structure in the equivalent nonlinear static analysis for cable breakage

646 events.

\subsection{Response envelopes for bridge girder}

The response envelopes along the bridge girder in terms of vertical bending moment, lateral bending

649 moment and torsional moment due to single-cable breakage are demonstrated in Figs. 15a, b and c,

650 respectively. It is found that the equivalent static analysis with a DAF of 2.0 generally provides smaller

651 values of both positive and negative bending moments and torsional moments with only a few exceptions.

652 It suggests that equivalent static analysis with a DAF of 2.0 for the cable loss event as defined by PTI

653 may not be able to capture the maximum responses on long-span bridge girders and a detailed nonlinear

654 dynamic analysis may be needed for the cable-breakage design.

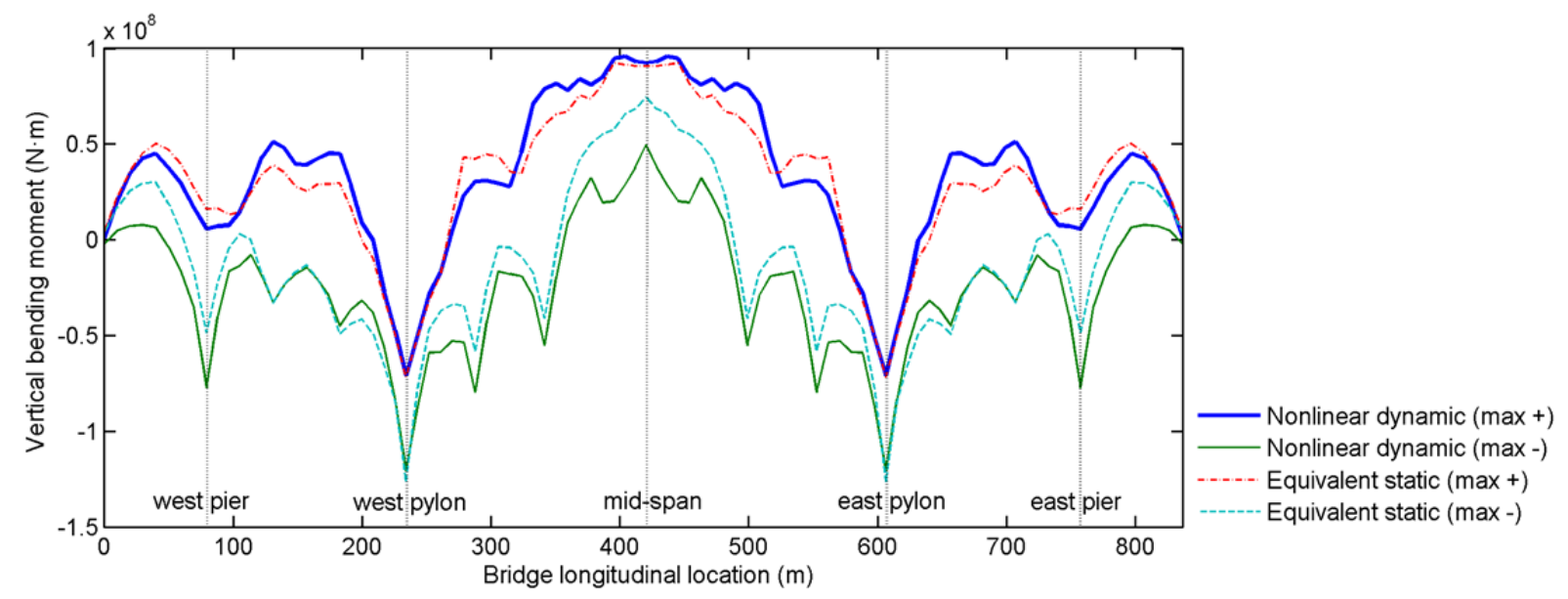

(a) Vertical bending moments 


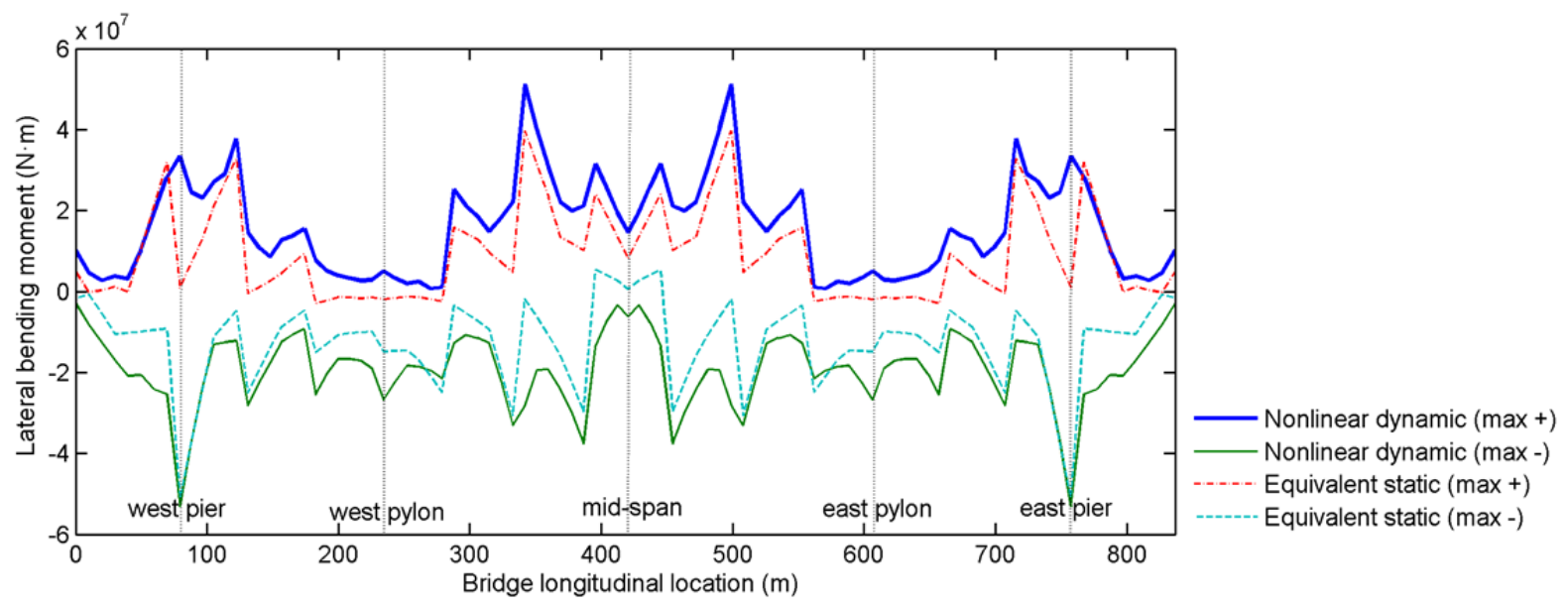

(b) Lateral bending moments

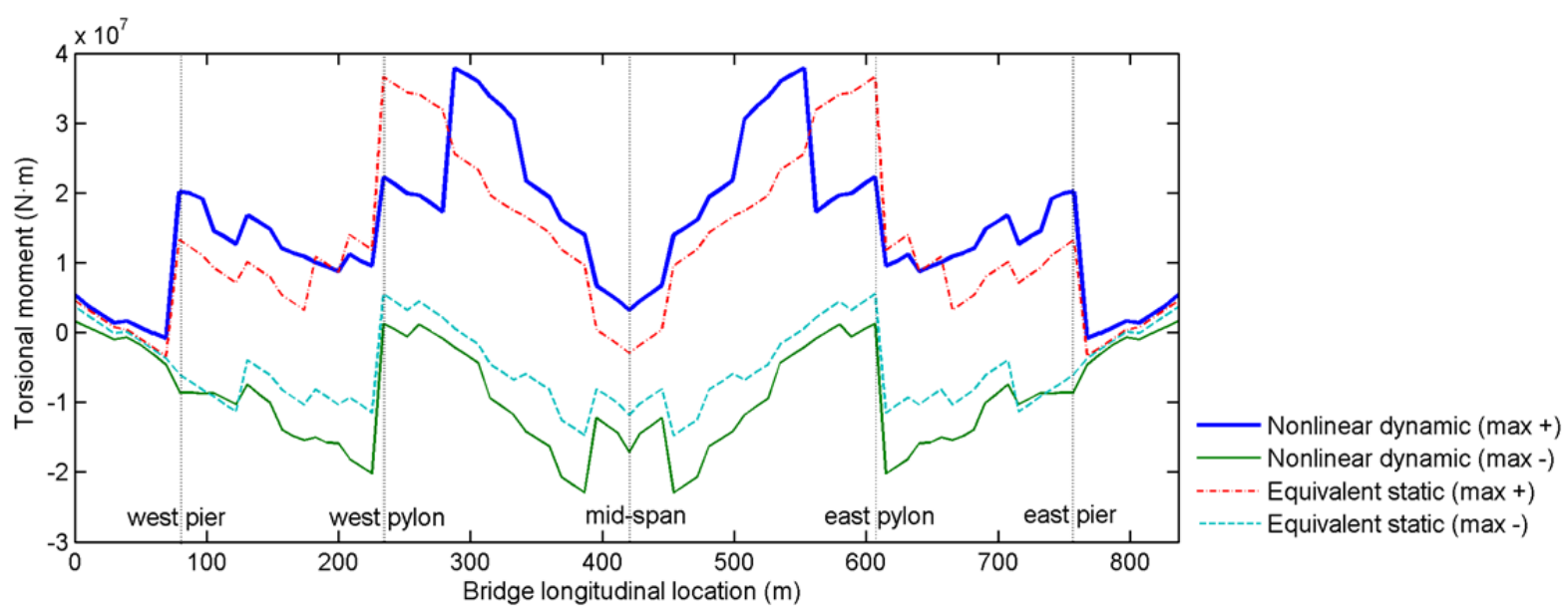

(c) Torsional moments

Fig. 15 Response envelopes of bridge girder using nonlinear dynamic and equivalent static analysis

\subsection{Response envelopes for bridge pylons}

Figs. 16a, b and $\mathrm{c}$ present the response envelopes along the south pylon column in terms of

664 longitudinal bending moment, transverse bending moment and torsional moment due to single-cable

665 breakage, respectively. It is found in Fig. 16a that the equivalent static analysis with a DAF of 2.0 will

666 give much smaller longitudinal bending moment envelopes than those from nonlinear dynamic analysis.

667 The transverse bending moment envelopes (Fig. 16b) from equivalent static analysis have relatively

668 similar trend to those from nonlinear dynamic analysis but with unsafe results. For the most part, the 
669 torsional moments obtained from nonlinear static analysis are smaller than those from nonlinear dynamic 670 analysis with a few exceptions, as shown in Fig. 16c. It is concluded that equivalent nonlinear static 671 analysis with a DAF of 2.0 will incur non-neglegible error in determining the response envelopes of 672 bridge pylon, and therefore is unsafe for the design of cable-stayed bridge pylons subjected to single673 cable loss when moderate wind $(20 \mathrm{~m} / \mathrm{s})$ and moderate traffic exist.
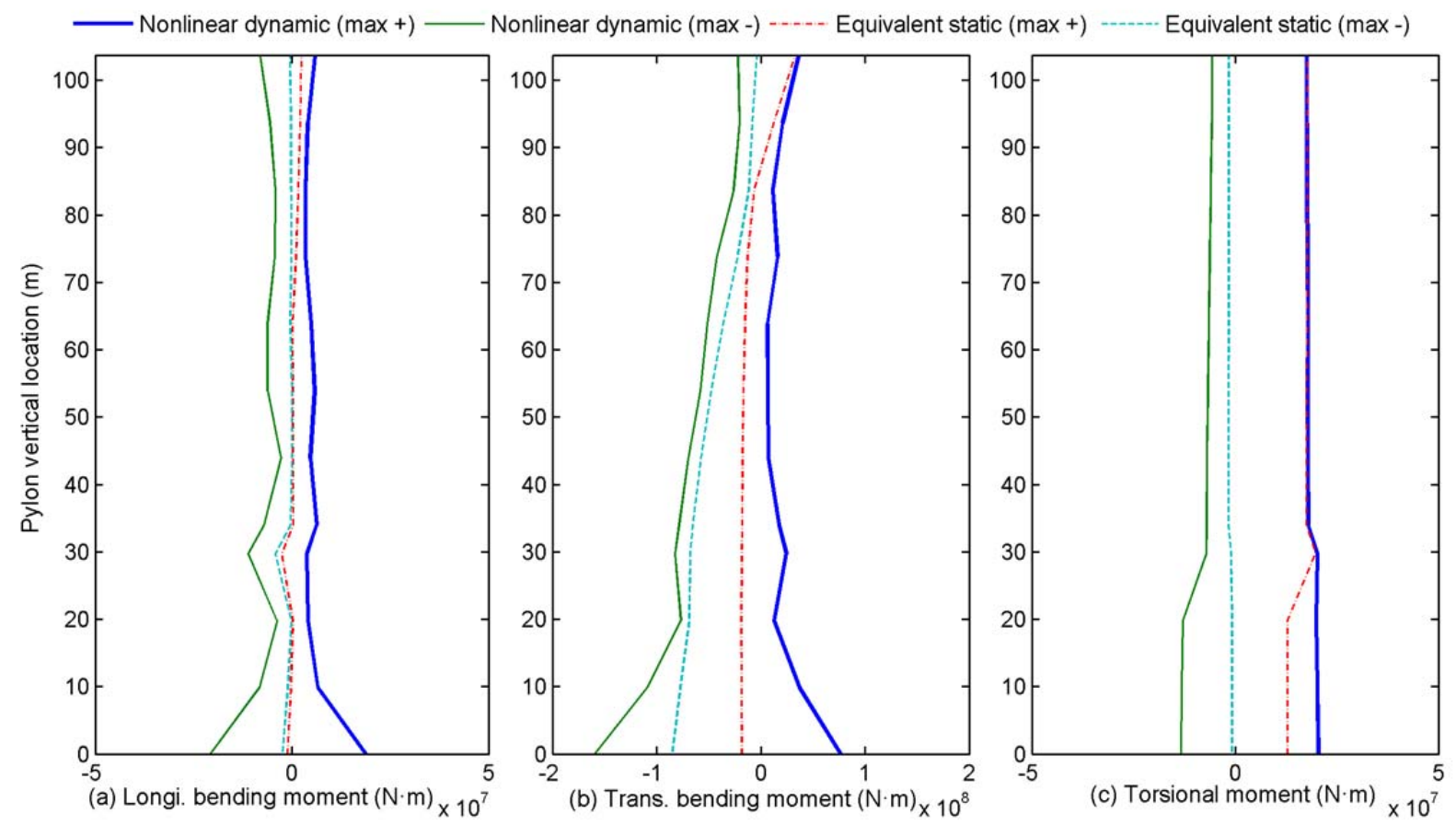

Fig. 16 Response envelopes of south pylon column using nonlinear dynamic and equivalent static

\subsection{Response envelopes for stay cables}

As shown in Fig. 17, the response envelopes in terms of tension forces of cables have similar shapes using both nonlinear dynamic and static analyses. Equivalent static analysis with a DAF of 2.0 is found unsafe for the design of Cable 1a-4a, although being safe for the design of Cable 5a and 6a. Considering cable arrangements can vary significantly among different cable-stayed bridges, bridge-specific investigation on the tension forces of the remaining cables during cable-loss events is felt needed. 


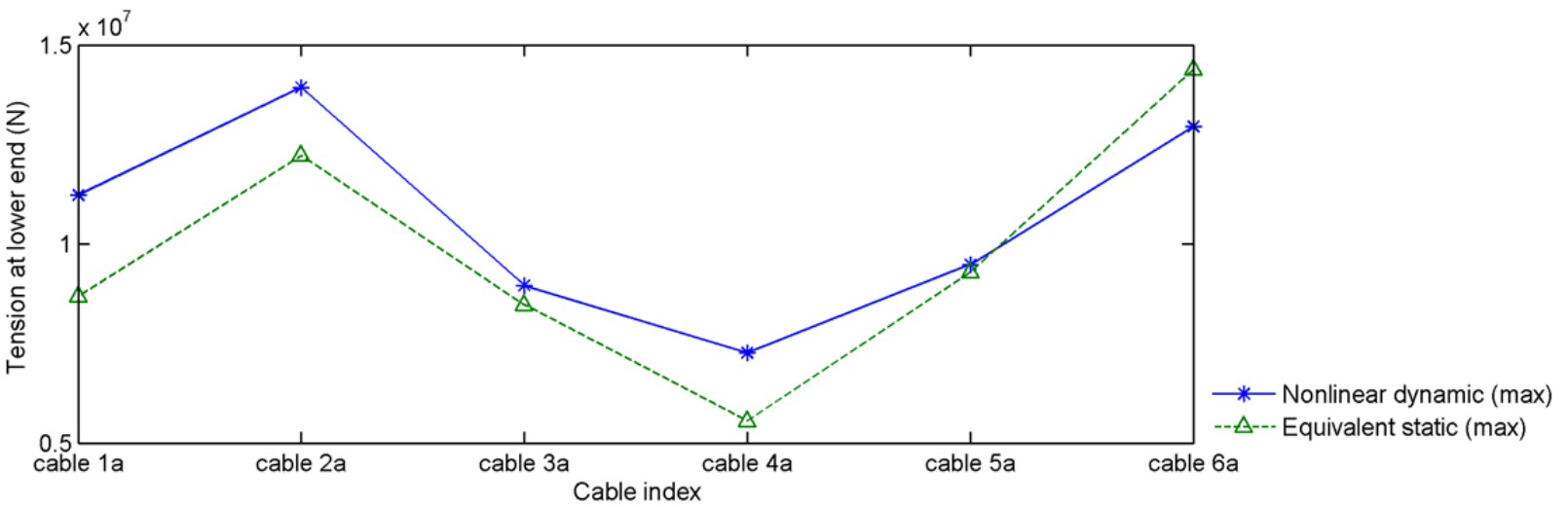

Fig. 17 Tension force envelopes for stay cables using nonlinear dynamic and equivalent static analysis

\section{Conclusions}

Single-cable breakage events have been comprehensively simulated on a representative long-span cable-stayed bridge with the newly proposed nonlinear dynamic methodology considering dynamic excitations from traffic and wind. Firstly, parametric studies on several critical variables during singlecable breakage events were conducted such as cable breakage duration, time-dependent cable area loss pattern, and the time instant of cable breakage occurrence and associated dynamic initial states. The internal forces/moments on bridge girder, pylons and remaining cables due to different single-cable breakage scenarios were then explored based on the nonlinear dynamic analysis results. Furthermore, the dynamic influence of stochastic traffic, wind excitations and their combined effects on the internal force and moments were studied. Due to the lack of relevant data, some assumptions had to be made in order to carry out the present study. These assumptions include the time duration and process of the cable breakage process and the driving behavior following the cable breakage events. Main findings drawn from the parametric studies of single-cable breakage cases are summarized as follows:

o Larger dynamic response will be induced in the breakage cases with shorter breakage durations when the breakage duration is less than the bridge fundamental period. Dynamic response is observed to become larger as the breakage process approaches abrupt loss process while it becomes smaller as the 
breakage process approaches linear loss process. Given the fact that rational characterization of the total breakage duration as well as the process of cable area loss is not yet available, uncertainties associated with these two factors are found necessary to be considered appropriately.

o Cable breakage can occur at any time instant on a vibrating bridge under service loads with different dynamic initial conditions. In both vertical and torsional directions, the maximum response will be achieved when the instantaneous bridge response under service loads reaches a local peak value, and start to move in an opposite direction coinciding with the direction of movement caused by the instantaneous unbalance as a result of the cable loss. The instantaneous unbalance and rebalance process of the bridge structure subjected to cable breakage may reinforce or suppress the existing various uncertainties based on the reliability theory.

715 o Compared with the vertical bending moments of bridge girder, the torsional moment of bridge girder, 716 the bending and torsional moments of bridge pylon columns are more likely to be influenced in a 717 cable breakage event. Tension forces of the remaining cables received least influence with relatively 718 smaller amplification factors during the cable breakage event.

719 o Cable breakage with simultaneous presence of busy stochastic traffic and wind will generally induce 720 larger internal force or moment along bridge girder, pylon and cables than those in the cases which do not consider the combined effects from traffic and wind. Fully coupled consideration of the bridgewind-stochastic traffic is found important to cable-breakage simulation of long-span bridges by capturing the extreme values of both pre-breakage and post-breakage responses.

o The breakage of a single cable on the prototype long-span cable-stayed bridge when service loads from stochastic traffic and wind loads are applied simultaneously will not cause the formation of plastic hinges on the bridge members, nor progressive collapse of the bridge structure. For the prototype long-span cable-stayed bridge being studied, it was found to be capable of withstanding the 
breakage of a single cable under service traffic and wind loads without reaching any strength limit state.

Finally, a comparison was made between the response envelopes in terms of bending and torsional moments of different bridge components from the nonlinear dynamic analysis and also the equivalent

732 nonlinear static analysis with a DAF of 2.0 as recommended by PTI. The results of the prototype bridge 733 show that the equivalent nonlinear static analysis with a DAF of 2.0 is unsafe for most of the moment 734 design of bridge girder, unsafe for the force design of some stay cables, and very unsafe and will incur 735 large errors for the design of bridge pylons. It should be noted, however, that some observations about the 736 results of using the PTI approaches may be specific to the bridge being studied and a more general 737 assessment about the PTI specification cannot be made until more bridges are investigated.

\section{Acknowledgement}

739 This research is supported by the National Science Foundation Grant CMMI-1335571, which is gratefully 740 acknowledged. The writers take sole responsibility for the views expressed in the paper, which may not 741 represent the opinions of the sponsor.

\section{References}

743 AASHTO. (2010). AASHTO LRFD bridge design specifications, Washington, DC.

744 ASCE. (2003). "Assessment of performance of vital long-span bridges in the United States." ASCE 745 subcommittee on performance of bridges, R. J. Kratky, ed., ASCE, Reston, VA.

746 Baker, C. J. (1986). "A simplified analysis of various types of wind-induced road vehicle accidents", 747 Journal of Wind Engineering and Industrial Aerodynamics, 22 (1986), 69-85.

748 Cai, J. G., Xu, Y. X., Zhuang, L. P., Feng, J. and Zhang, J. (2012). “Comparison of various procedures for 749 progressive collapse analysis of cable-stayed bridges." Journal of Zhejiang University-science A (Applied physics and Engineering), 2012 13(5), 323-334. 
Chen, S. R., Cai, C.S. and Wolshon, B. (2009). "From normal operation to evacuation: single-vehicle safety under adverse weather, topographic and operational conditions." Natural Hazards Review, ASCE, $10(2), 68-76$.

Chen, S. R. and Wu, J. (2010). "Dynamic performance simulation of long-span bridge under combined loads of stochastic traffic and wind", Journal of Bridge Engineering, 15(3), 219-230.

Chen, S. R. and Wu, J. (2011). "Modeling stochastic live load for long-span bridge based on microscopic traffic flow simulation”, Computer \& Structures, 89, 813-824. MATLAB version R2013b [Computing language]. MathWorks, Natick, MA.

Mozos, C.M. and Aparicio A.C. (2010a). "Parametric study on the dynamic response of cable stayed bridges to the sudden failure of a stay, part I: bending moment acting on the deck." Engineering Structures, 32(2010), 3328-3300.

762 Mozos, C.M. and Aparicio, A.C. (2010b). "Parametric study on the dynamic response of cable stayed 763 bridges to the sudden failure of a stay, part II: bending moment acting on the pylons and stress on the 764 stays.” Engineering Structures, 32(2010), 3301-3312.

765 Mozos, C.M. and Aparicio, A.C. (2011). "Numerical and experimental study on the interaction cable 766 structure during the failure of a stay in a cable stayed bridge." Engineering Structures, 33(8), p. 23307672341.

768 National Transportation Safety Board (NTSB) (2007). "Collapse of I-35W Highway Bridge Minneapolis, 769 Minnesota", Accident report, NTSB/HAR-08/03, Washington, D.C..

770 Post-Tensioning Institute (PTI) (2007). Recommendations for Stay Cable Design, Testing and Installation. 771 Fifth edition. Cable-Stayed Bridges Committee, Phoenix, USA.

772 Ruiz-Teran, A. and Aparicio, A. (2009). "Response of under-deck cable-stayed bridges to the accidental 773 breakage of stay cables”, Engineering Structure, 31 (2009), 1425-1434.

774 Wolff, M. and Starossek, U. (2010). "Cable-loss analyses and collapse behavior of cable-stayed bridges." 775 IABMAS 2010. The Fifth International Conference on Bridge Maintenance, Safety and Management, 776 July 2010, Philadelphia, USA. 
777 Zhou, Y. F. and Chen, S. R. (2014a). "Time-progressive dynamic assessment of abrupt cable-breakage 778 events on cable-stayed bridges." Journal of Bridge Engineering, ASCE, 19(2), 159-171.

779 Zhou, Y. F. and Chen, S. R. (2014b). "Framework of fully-coupled nonlinear dynamic simulation of long780 span cable-stayed bridges subjected to cable-loss incidents." Journal of Structural Engineering, ASCE 781 (under revision for re-review).

782 Zhou, Y. F. and Chen, S. R. (2015). "Fully coupled driving safety analysis of moving traffic on long-span 783 bridges subjected to crosswind", Journal of Wind Engineering and Industrial Aerodynamics, 143, 1-18.

784 Zoli, T. and Woodward, R. (2005). "Design of Long Span Bridges for Cable Loss." International 785 Association for Bridge and Structural Engineering (IABSE) Symposium Report, Lisbon 2005: Structure 786 and Extreme Events, pp 17-25 (9). 